\title{
Prospect Dynamics and Loss Dominance
}

\author{
Ryoji Sawa and Jiabin $\mathrm{Wu}^{*}$
}

August 30, 2018

\begin{abstract}
This paper investigates the role of loss-aversion in affecting the long-run equilibria of stochastic evolutionary dynamics. We consider a finite population of loss-averse agents who are repeatedly and randomly matched to play a symmetric two-player normal form game. When an agent revises her strategy, she compares the payoff from each strategy to a reference point. Under the resulting dynamics, called prospect dynamics, risk-dominance is no longer sufficient to guarantee stochastic stability in $2 \times 2$ coordination games. We propose a stronger concept, loss-dominance: a strategy is loss-dominant if it is risk-dominant and a maximin strategy. In $2 \times 2$ coordination games, the state where all agents play the loss-dominant strategy is uniquely stochastically stable under prospect dynamics for any degree of loss-aversion and all types of reference points. For symmetric two-player normal form games, a generalized concept, generalized loss-dominance, gives a sufficient condition for stochastic stability under prospect dynamics.
\end{abstract}

Keywords: Evolutionary game theory, Behavioral game theory, Equilibrium selection, Lossaversion, Prospect dynamic, Loss-dominance, Risk-dominance, Half-dominance, Maximin.

JEL Code: C72, C73.

*Sawa: Department of Policy and Planning Sciences, University of Tsukuba, email: rsawa@sk.tsukuba.ac.jp. Wu: Department of Economics, University of Oregon, email: jwu5@uoregon.edu. Please direct correspondence to either author. 


\section{Introduction}

Loss-aversion captures the tendency of people being more sensitive to losses than to gains. It is a type of reference-dependent preference which describes a person's assessment of choices by comparing the corresponding outcomes to a reference point. Pioneered by Kahneman and Tversky (1979)'s seminal work on prospect theory, loss-aversion has been widely accepted as a common psychological departure from standard expected utility theory that can affect individual decisions. For example, Köszegi and Rabin (2006) applied loss-aversion to explain some well-known phenomena in individual consumption behavior and daily labor supply decisions. ${ }^{1}$

However, little is known about how and to what extent loss-aversion affects the long-run behavior of people in society. Answering this question would enable us to better understand societal phenomena such as the emergence of social conventions, norms and traditions. In this paper, we attempt to answer this question via an evolutionary approach. We employ stochastic stability analysis as the main analytical tool for two reasons. 1) It offers models to examine the long-run equilibrium of games played by boundedly rational agents. Hence, it is natural to incorporate psychological biases such as loss aversion into evolutionary models. 2) It often gives sharp equilibrium selection results when there are multiple Nash equilibria. We compare the long-run equilibrium for settings where the value of a strategy equals the probability-weighted average of its outcomes with the long-run equilibrium for settings where the value of a strategy is affected by loss aversion. This allows us to examine whether the predictions coincide or differ under the two theories, expected utility theory and prospect theory.

We consider a finite population of loss-averse agents who are recurrently and randomly matched to play a symmetric two-player normal form game. ${ }^{2}$ In each discrete-time period, one agent is given an opportunity to revise her strategy. When an agent receives a revision opportunity, she compares the payoff of each strategy to her reference point and switches to the one with the highest value (with probability close to one). We call the evolutionary dynamics generated by such loss-averse agents the prospect dynamics.

We analyze stochastic stability of the population states under prospect dynamics. To make a more relevant comparison to the extant literature, we mainly focus on $2 \times 2$ coordination games and characterize long-run equilibria of the games. Our results provide a new insight to evolutionary

\footnotetext{
${ }^{1}$ See also Thaler (1980), Knetsch and Sinden (1984), Samuelson and Zeckhauser (1988), among many others.

${ }^{2}$ We assume that agents are identically loss-averse in most part of the paper. We relax the assumption and consider heterogeneity in the degree of loss-aversion in Section 7.1.
} 
equilibrium selection in coordination games. Kandori et al. (1993) and Young (1993) showed that a risk-dominant equilibrium is uniquely selected in a population of agents who do not have lossaverse preferences. In contrast to those studies, we find that risk-dominance is no longer sufficient to guarantee stochastic stability under prospect dynamics. Moreover, even if an equilibrium is both payoff-dominant and risk-dominant, it may not be always selected in the long run. Therefore, we propose a stronger concept, loss-dominance: a strategy is loss-dominant if it is both the riskdominant strategy (Harsanyi and Selten (1988)) and the maximin strategy (von Neumann and Morgenstern (1944)). The strategy is less risky and is associated with the largest payoff in the worst case scenario. This concept captures the tendency of people to avoid not only risks but also losses. Our main result, Theorem 3.2, shows that the state in which all agents play a loss-dominant strategy is uniquely stochastically stable under prospect dynamics for any degree of loss-aversion and for any type of reference points.

The assumption of the $2 \times 2$ coordination game is relaxed in Section 4 , where we generalize the concept of loss-dominance for symmetric two-player normal form games. A strategy is a generalized loss-dominant strategy if it is half-dominant and a globally pairwise maximin strategy, an extension of the notion of maximin. This new concept captures the essence of the concept of loss-dominance. Our second main result (Theorem 4.4) shows that, in a class of symmetric two-player normal form games, the generalized loss-dominance guarantees stochastic stability under prospect dynamics for any degree of loss-aversion and for any type of reference points.

We then consider two models with endogenous reference points that naturally arise in a game theoretical context: The first model is called the social comparison model. Pioneered by the research of Festinger (1954), social psychologists have found that social comparison often serves as a driving force in determining a person's beliefs and decisions. ${ }^{3}$ More specifically, people derive extra utility when they believe that they are doing better than other individuals, while they incur disutility when they believe that they are falling behind others. In this context, the social average payoff can serve as a natural reference point for an agent to evaluate whether each possible outcome she would get is a success or a failure comparing to the other agents.

The second model is called the status-quo bias model. Status-quo bias is one of the most wellstudied psychological biases (Samuelson and Zeckhauser (1988)), and it may appear in a variety of decision-making situations. For example, it offers an explanation for the endowment effect found by Thaler (1980). Experimental studies have shown that peoples' decisions are biased toward adhering

\footnotetext{
${ }^{3}$ In economics, the ideas of inequality aversion (Fehr and Schmidt (1999)) and status competition (Veblen (1899), Hopkins and Kornienko (2009)) also capture people's tendency to make comparison with others.
} 
to status-quo choices, and it is as if people were to take the status-quo as a reference point. Hence, this bias can also be modeled by loss aversion (see Kahneman et al. (1991), for example). In a dynamic context, an agent may regard the payoff obtained in the latest game she played as her status-quo, and may use it as her reference point.

Our main result implies that loss-dominance is a sufficient condition for stochastic stability in $2 \times 2$ coordination games under these two models. However, necessary conditions differ in the two models. We are interested to which extent they differ. To this end, we investigate games that have no loss-dominant strategy, i.e., the risk-dominant strategy differs from the maximin strategy. For each model, we characterize the precise condition for a state to be stochastically stable for all degrees of loss-aversion. We find that the state in which all agents play the risk-dominant strategy will be stochastically stable if the largest payoff associated with the risk-dominant strategy is larger than a threshold. In other words, the payoff advantage of the strategy in the best case scenario compensates the perceived loss brought by the strategy in the worst case scenario. We call such a strategy the compensation-attractive strategy.

Finally, we extend the model to allow agents to have diminishing sensitivity to gains and losses, which is another important element of prospect theory besides loss-aversion. We find that lossdominance still guarantees stochastic stability as long as agents' degree of diminishing sensitivity to gains/losses is not sufficiently small.

Related studies in the literature on stochastic evolutionary games are Norman (2009) and Sawa (2015), both examined stochastic stability with behavioral biases that are similar to what we consider in this paper. Norman (2009) introduced a switching cost for strategy revisions, which can be viewed as a status-quo bias. Sawa (2015) considered stochastic stability in bargaining games with players obeying prospect theory. Our model differs from theose studies in two aspects. First, we do not restrict reference points to one particular type, but provide a general condition that guarantees stochastic stability for all types of reference points. Second, we consider models with endogenous reference points, while those studies assume exogenous switching costs or reference points. ${ }^{4}$ Another related study is a companion paper of ours, Sawa and Wu (2018), that addresses

\footnotetext{
${ }^{4}$ This paper also shares some similarities with a few works in the literature of learning. Sarin and Vahid (1999) provided an adaptive learning foundation for maximin strategies in an uncertain world. Börgers and Sarin (2000) considered a reinforcement learning model with endogenous aspiration level, and showed that as the aspiration level increases, a decision maker's incentive can be distorted from reaching the efficient outcome. The main differences between our paper and these works are that we consider strategic interaction and provide a psychological foundation for the maximin strategy.
} 
(universal) stochastic stability for a broad class of preferences including loss-averse preferences with any degree of diminishing sensitivity. In contrast to the results here, Sawa and Wu (2018) show that for such a class of preferences, an equilibrium must be not only loss-dominant but also payoff-dominant for it to be stochastically stable.

Many recent papers contribute to the understanding of stochastic evolutionary dynamics and equilibrium selection problem in coordination games. See for example, Maruta (2002), Blume (2003), Peski (2010), Sandholm (2010), Neary (2012), Staudigl (2012), Staudigl and Weidenholzer (2014), Kreindler and Young (2013), Lim and Neary (2016), Bilancini and Boncinelli (2016), Sandholm and Staudigl (2016), Mäs and Nax (2016), Hwang and Newton (2017), Hwang and Rey-Bellet (2017) and Newton (2018a), among many others. See Newton (2018b) for an excellent survey. Among these works, Bilancini and Boncinelli (2016) consider symmetric two-player, two-strategy games for a class of choice rules including the condition-dependent mistake model, in which the error probability negatively depends on the payoff earned in the last period. In this respect, there is some similarity to the status-quo bias model in this paper in which agents use the last earned payoff as a reference point. They find that the state where every player plays the maximin strategy is stochastically stable for some class of games if players adopt the condition-dependent mistake model and rematch every period. In addition, Mäs and Nax (2016) find in their experiment that a payoff decrease in the previous period would induce higher deviation rates from myopic best response behavior, which indicates that subjects' choices are sensitive to past payoff losses.

This paper is also related to several studies in the experimental literature. Cachon and Camerer (1996), Rydval and Ortmann (2005), Feltovich (2011), and Feltovich et al. (2012) studied whether subtracting a constant payoff from people's game payoffs would affect their behavior in games. They found evidence that people are sensitive to outcomes being framed as losses. This together with our theoretical results would suggest that long-run equilibria of a society can be affected by its members' cognitive biases.

The paper is organized as follows. Section 2 introduces the model. Section 3 defines lossdominance, delivers the main result on stochastic stability in $2 \times 2$ coordination games. Section 4 extends the main result to symmetric two-player normal form games. Section 5 studies prospect dynamics in two models of endogenous reference points: 1) the social comparison model, and 2) the status-quo bias model. Section 6 extends our main results to reference-dependent preferences combined with diminishing sensitivity to gains/losses. We discuss the robustness of our results and consider a few extensions of the model in Section 7. Section 8 concludes. 


\section{The Model}

\subsection{The Population Game and Its Dynamics}

We consider a dynamic process in which $n<\infty$ identical loss-averse agents are repeatedly and randomly matched to play a coordination game in discrete time. We restrict our attention to $2 \times 2$ symmetric coordination games with strategy space $S=\{0,1\}$. The monetary returns received from the game are illustrated in Table $1 .^{5}$ The state of the process is described by the current fraction of agents playing strategy 1 . Let $X \equiv\left\{0, \frac{1}{n}, \frac{2}{n}, \ldots, 1\right\}$ denote the state space, and $x \in X$ denote the current state. $x$ is observed by every agent.

Table 1: $2 \times 2$ Coordination Game (Monetary Returns)

Player 2

\begin{tabular}{cc|c|c|}
\multicolumn{1}{c}{0} & \multicolumn{1}{c}{0} \\
\cline { 3 - 4 } Player 1 & 0 & $a_{00}, a_{00}$ & $a_{01}, a_{10}$ \\
\cline { 3 - 4 } & 1 & $a_{10}, a_{01}$ & $a_{11}, a_{11}$ \\
\cline { 3 - 4 } & & &
\end{tabular}

The monetary outcomes satisfy $a_{00}, a_{01}, a_{10}, a_{11}>0, a_{00}>a_{10}$ and $a_{11}>a_{01}$. A pure strategy profile $(s, s)$ is said to be risk-dominant if $a_{s 0}+a_{s 1}>a_{s^{\prime} 0}+a_{s^{\prime} 1}$ for $s^{\prime} \neq s$, and we also call such strategy $s$ risk-dominant. Similarly, strategy profile $(s, s)$ and strategy $s$ are said to be payoffdominant if $a_{s s}>a_{s^{\prime} s^{\prime}}$ for $s^{\prime} \neq s$. We will shortly assume that the utility function is strictly increasing in monetary returns. Thus, $(0,0)$ and $(1,1)$ are strict Nash equilibria. The set of pure strategy Nash equilibria remains unchanged throughout the paper although we consider different models of reference points and various degrees of loss aversion.

In the dynamic process, each agent occasionally reconsiders her choice of strategy, using myopic best response rules. Myopic best responses for an agent are not determined by monetary returns but by her perceived utilities, which will be introduced in Section 2.2. Their myopic choice rules are assumed to be perturbed by stochastic noise. The choice rule, the dynamics and the evolutionary selection principle will be introduced in Section 2.3.

\footnotetext{
${ }^{5}$ Note that Shalev (2000) and Köszegi and Rabin (2006) proposed loss-aversion utility functions defined over von Neumann-Morgenstern utility functions, which are different from the conventional way of modeling loss-aversion over monetary outcomes initiated by Kahneman and Tversky (1979). In this paper, we follow Kahneman and Tversky (1979), but we emphasize that replacing the monetary outcomes with von Neumann-Morgenstern utility functions would not change our main results.
} 


\subsection{Loss Aversion and Reference-Dependent Utility}

Agents' preferences are reference-dependent. Each agent has a reference point, which determines whether she perceives the monetary return from the game as a gain or a loss. The reference point of an agent can be endogenously determined. A model of reference points is given by a function $r(\cdot)$ explained below. ${ }^{6}$

If a reference point is endogenous, then it is assumed to depend on her reference strategy (it could be her current strategy, a strategy she is considering switching to, and so forth), her opponent's strategy as well as the population state. The reference point of an agent $i$ is given by a function $r\left(s_{i}^{r}, \hat{s}_{i}, x\right):\{0,1\} \times\{0,1\} \times[0,1] \rightarrow \mathbb{R}$, where $s_{i}^{r}$ is agent $i$ 's reference strategy, $\hat{s}_{i}$ is her (last) matched opponent's strategy, and $x$ is the population state. If a reference point is exogenous, then it is constant over time, $r\left(s_{i}^{r}, \hat{s}_{i}, x\right)=r$ for some $r \in \mathbb{R}$.

Example 1 (social comparison). A person's reference point can be determined by other people's achievements. People may evaluate their outcomes relative to the social average of monetary payoffs, that is, the reference point is given by

$$
r\left(s_{i}^{r}, \hat{s}_{i}, x\right)=(1-x)\left((1-x) a_{00}+x a_{01}\right)+x\left((1-x) a_{10}+x a_{11}\right) .
$$

We examine a model of such social comparisons in Section 5.1.

Following Kahneman and Tversky (1979), we model the value function of a loss-averse agent who anticipates receiving the payoff $a_{s s^{\prime}}$ as follows: ${ }^{7}$

$$
v\left(a_{s s^{\prime}}, r\left(s_{i}^{r}, \hat{s}_{i}, x\right)\right)= \begin{cases}\left(a_{s s^{\prime}}-r\left(s_{i}^{r}, \hat{s}_{i}, x\right)\right) & \text { if } a_{s s^{\prime}}-r\left(s_{i}^{r}, \hat{s}_{i}, x\right) \geq 0, \\ \lambda\left(a_{s s^{\prime}}-r\left(s_{i}^{r}, \hat{s}_{i}, x\right)\right) & \text { otherwise }\end{cases}
$$

where $\lambda \geq 1$.

The value function indicates that the agent derives positive value from gains and negative value from losses. The degree of loss-aversion is measured by $\lambda$. If $\lambda>1$, then she dislikes losses more than she likes same-sized gains. Given the value function, an agent's reference-dependent utility of

\footnotetext{
${ }^{6}$ For heterogeneous reference points, which we will discuss in Section 7.1, a model of reference points is given by a tuple of functions, $\left\{r_{i}(\cdot)\right\}_{i \in N}$.

${ }^{7}$ For now, we assume that agents are risk-neutral. In Section 6, we incorporate risk-attitudes (diminishing sensitivity to losses/gains) to the model and show that our main results are robust as long as the degree of agents' diminishing sensitivity to losses/gains is not sufficiently small.
} 
choosing strategy $s \in\{0,1\}$ is given by ${ }^{8}$

$$
u_{s}\left(x, r\left(s_{i}^{r}, \hat{s}_{i}, x\right)\right)=(1-x) v\left(a_{s 0}, r\left(s_{i}^{r}, \hat{s}_{i}, x\right)\right)+x v\left(a_{s 1}, r\left(s_{i}^{r}, \hat{s}_{i}, x\right)\right) \text {, }
$$

which is the expectation of her value function. The probability weights are objective and equal to the probabilities of strategies that this agent faces. ${ }^{9}$

\subsection{Prospect Dynamic and Stochastically Stable States}

The agents are recurrently and randomly matched to play the coordination game in Table 1. For simplicity in terms of presenting the model, we allow self-matching in the matching protocol and assume that agents follow a simple decision rule under which they do not take into account the effect of strategy change on their utilities when revising strategies. However, we emphasize that our results are robust to different matching protocols and decision rules. ${ }^{10}$

In each period $t \in\{1,2, \ldots\}$, one agent is randomly chosen and given an opportunity to revise her strategy. Given the revision opportunity, an agent is matched with another randomly selected agent to play the game, and then revise her strategy probabilistically according to the following simple noisy best response revision protocol:

$$
\begin{aligned}
& \rho_{01}\left(x, r_{0}\right)=\sigma^{\eta}\left(u_{1}\left(x, r_{0}\right)-u_{0}\left(x, r_{0}\right)\right), \\
& \rho_{10}\left(x, r_{1}\right)=\sigma^{\eta}\left(u_{0}\left(x, r_{1}\right)-u_{1}\left(x, r_{1}\right)\right),
\end{aligned}
$$

where $\sigma^{\eta}: \mathbb{R} \rightarrow[0,1], r_{s}=r\left(s, \hat{s}_{i}, x\right)$, and $\eta>0$ denotes the noise level. $\rho_{01}\left(x, r_{0}\right)$ denotes the probability that an agent playing strategy 0 switches to strategy $1 . \rho_{10}\left(x, r_{1}\right)$ is the probability that an agent playing strategy 1 switches to strategy $0 . \rho_{01}\left(x, r_{0}\right)$ and $\rho_{10}\left(x, r_{1}\right)$ are functions of the utility change that the agent expects from switching strategy, i.e., $u_{1}\left(x, r_{0}\right)-u_{0}\left(x, r_{0}\right)$ and $u_{0}\left(x, r_{1}\right)-u_{1}\left(x, r_{1}\right)$ respectively. Note that the revising agent is matched with another agent, whose strategy becomes the (last) matched opponent's strategy for the revising agent. We assume that

\footnotetext{
${ }^{8}$ In Köszegi and Rabin (2006), the utility function of a loss-averse consumer depends on both the absolute consumption level and the value of gains/losses. We argue that adding the absolute payoff level into the agent's utility function in our model would not change the main results.

${ }^{9}$ The probabilities people assign to stochastic events might be subjective and be influenced by loss-aversion (see Tversky and Kahneman (1992)). In our model, we assume that all agents can observe the population state and evaluate outcomes with objective probabilities.

${ }^{10}$ Please refer to Section 7.2 for a detailed discussion.
} 
$\sigma^{\eta}(\cdot)$ becomes the best response protocol in the limit of the small noise level, that is,

$$
\lim _{\eta \rightarrow 0} \sigma^{\eta}(a)= \begin{cases}1 & \text { if } a>0 \\ 0 & \text { if } a<0\end{cases}
$$

We call the dynamic generated by (2) and (3) prospect dynamic. In particular, we adopt the widely used protocol, best response with mutations (BRM) from Kandori et al. (1993), which is defined by

$$
\sigma^{\eta}(a)= \begin{cases}1-\exp \left(-\eta^{-1}\right) & \text { if } a>0 \\ \exp \left(-\eta^{-1}\right) & \text { if } a<0\end{cases}
$$

Consider an agent playing strategy 0. When she is given a revision opportunity, she switches to strategy 1 with probability $\rho_{01}\left(x, r_{0}\right)$. Similarly, when an agent playing strategy 1 is given a revision opportunity, she switches to strategy 0 with probability $\rho_{10}\left(x, r_{1}\right)$. This model defines a Markov chain $\left\{X_{t}^{n}\right\}$ on the discrete state space $X .{ }^{11}$ Observe that each $x \in\{0,1\} \subset X$ corresponds to a pure-strategy Nash equilibrium of the game.

We examine the stationary distribution $\mu$ of the Markov chain when the noise level approaches zero and the population size is sufficiently large. We say that state $x \in X$ is stochastically stable if $x$ has a positive mass in the limiting stationary distribution for all sufficiently large $n$,

$$
\lim _{\eta \rightarrow 0} \mu(x)>0
$$

In the subsequent sections, we employ the 'mutation counting' technique from Kandori et al. (1993) and Young (1993) to characterize stochastically stable states. The set of states for which strategy $s$ is unique best response is called the basin of attraction of the all- $s$ state. Theorem 1 of Sandholm (2010) showed that, for all sufficiently large $n$, the mutation counting technique reduces to examining which strategy has a greater basin of attraction on the continuous interval $[0,1]$. That is, in order to prove that the all-0 state is uniquely stochastically stable, it suffices to show that strategy 0 is the unique best response for all $x \in[0,1 / 2]$.

Remark 2.1. The simple noisy best response revision protocol we specify assumes that agents regard strategy $s$ as the most successful option if $u_{s}\left(x, r_{s}\right) \geq u_{s^{\prime}}\left(x, r_{s^{\prime}}\right)$ for $s^{\prime} \neq s$ and they try to "imitate success" by switching to $s$ with a probability going to 1 in the limit of the small noise

\footnotetext{
${ }^{11}$ Rigorously speaking, a state should include the profile of agents' reference strategies, because $r\left(s, \hat{s}_{i}, x\right)$ depends on it. However, for the examined behavioral models in Sections 3, 4, 5 and 6, the process only depends on $X$.
} 
level. Rigorously speaking, the notion of best response should take into account the effect of strategy change, see Kandori and Rob (1995) and Sandholm (1998). For example, in a $2 \times 2$ game, strategy 0 is a best response for agents playing 0 if $u_{0}\left(x, r_{0}\right) \geq u_{1}\left(x-\frac{1}{n}, r_{1}\right)$, and it is a best response for agents playing 1 if $u_{0}\left(x-\frac{1}{n}, r_{0}\right) \geq u_{1}\left(x, r_{1}\right)$. See Section 7.2 for a discussion on a decision rule in which agents take into account the effect of strategy change.

\section{Loss-dominance and its stochastic stability}

\subsection{Loss Dominance}

We propose a new concept called loss-dominance which combines two well-studied concepts: riskdominance and maximin strategy. ${ }^{12}$

Definition 3.1. Strategy $s \in\{0,1\}$ is a loss-dominant strategy if for $s^{\prime} \in\{0,1\}, s^{\prime} \neq s$,

(1) $a_{s 0}+a_{s 1}>a_{s^{\prime} 0}+a_{s^{\prime} 1}$,

(2) $\min \left\{a_{s 0}, a_{s 1}\right\} \geq \min \left\{a_{s^{\prime} 0}, a_{s^{\prime} 1}\right\}$.

A population state in which every agent is playing the loss-dominant strategy $s \in\{0,1\}$ (the all-s state) is a loss-dominant equilibrium.

The first condition in Definition 3.1 states that a loss-dominant strategy must be the riskdominant strategy. The second one states that it must also be the (weak) maximin strategy. Hence, loss-dominance captures not only people's reluctance to take risks but also the tendency to avoid losses, particularly the loss occurred in the worst case scenario. Observe that a sufficient condition for loss-dominance is that a strategy $s$ is risk-dominant but not payoff-dominant, i.e., $a_{s s}+a_{s s^{\prime}}>$ $a_{s^{\prime} s}+a_{s^{\prime} s^{\prime}}$ and $a_{s s}<a_{s^{\prime} s^{\prime}} \cdot{ }^{13}$

\subsection{Stochastic stability of loss-dominant equilibria}

For the sake of simplicity, we assume that all agents adopt the same model of reference points throughout the paper. However, the assumption can be readily relaxed, which will be discussed in Section 7.1. The next theorem shows that loss-dominance is a solution concept that passes the

\footnotetext{
${ }^{12}$ Similarly to experimental studies, e.g. Battalio et al. (2001) and Schmidt et al. (2003), we define those criteria in terms of (usually observable) monetary payoffs. Our intention is to examine if such criteria on monetary payoffs guarantee stochastic stability despite the effect of reference-dependent preferences.

${ }^{13}$ We thank a referee for this observation. Example 2 offers a graphic illustration.
} 
stochastic stability test under loss-averse preferences with any degree of loss-aversion. Most of the proofs in this paper are relegated to the Appendix.

Theorem 3.2. The all-s state is uniquely stochastically stable for all models of reference points and all $\lambda \geq 1$ if and only if strategy $s$ is loss-dominant.

The rationale behind Theorem 3.2 is as follows. Without loss of generality, let strategy 0 be the loss-dominant strategy. Note that being the maximin strategy implies that $a_{10}$ must be the smallest among $a_{00}, a_{01}, a_{10}$, and $a_{11}$. When $r>\max _{i, j \in S} a_{i j}$ or $r \leq a_{10}$, the payoffs are either all viewed as gains or all viewed as losses. Hence, being the risk-dominant strategy guarantees that the all-0 state is uniquely stochastically stable as in Kandori et al. (1993) and Young (1993). When $r \in\left[a_{10}, \max _{i, j \in S} a_{i j}\right], a_{10}$ is regarded as a loss and the loss associated with $a_{10}$ is the largest among all potential losses associated with the outcomes. Therefore, when the degree of loss-aversion is high, agents tend to avoid choosing strategy 1. This makes the basin of attraction of the all-0 state larger than the all-1 state. Hence, the all-0 state is uniquely stochastically stable. On the other hand, when the degree of loss-aversion is low, being risk-dominant ensures that the all-0 state is uniquely stochastically stable. Since agents' utilities from a loss are linear in the degree of loss-aversion $\lambda$, the all-0 state being uniquely stochastically stable for sufficiently small and large $\lambda$ implies that the same holds for all degrees of loss-aversion.

Payoff-dominance, risk-dominance, and maximin are well-known equilibrium selection principles for coordination games. Our result suggests that risk-dominance and maximin together play an important role in determining stochastically stable states for loss-averse agents. For smaller degrees of loss aversion, risk-dominance plays a major role in selecting equilibrium as in Kandori et al. (1993) and Young (1993). While for larger degrees of loss aversion, maximin becomes the dominant force in equilibrium selection. An interesting implication is that an equilibrium that is both payoff-dominant and risk-dominant can fail to be stochastically stable when agents are (highly) loss-averse. The following example provides a graphic illustration for the relationship between payoff-dominance, risk-dominance and maximin.

Example 2. Consider a coordination game with $a_{01}=0$ and $a_{11}=1$. In Figure 1, we put $a_{10}$ on the $x$-axis and $a_{00}$ on the $y$-axis. Since we are considering a coordination game, all feasible pairs of $\left(a_{10}, a_{00}\right)$ should be above the 45 degree dotted line through the origin. Strategy 0 is the payoff-dominant strategy if $a_{00}>a_{11}=1$, which is satisfied by all payoff profiles above the "PD" line. Strategy 0 is the risk-dominant strategy if $a_{00}-a_{10}>a_{11}-a_{01}=1$, which is satisfied by all profiles above the "RD" line. Finally, it is maximin if $a_{10}<0$, which is to the left of the $y$-axis. 


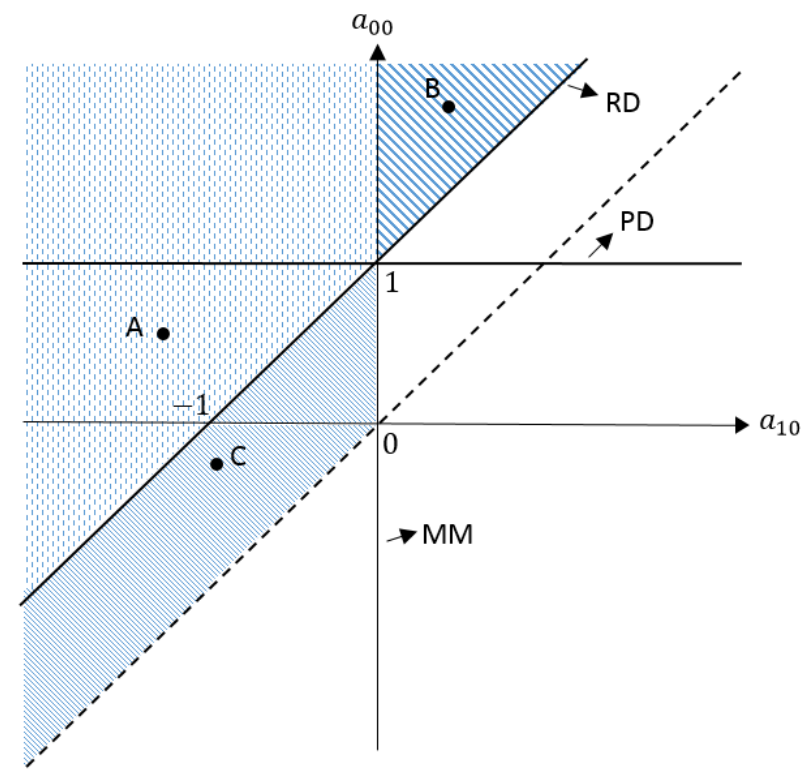

Figure 1: The relationship between payoff-dominance, risk-dominance and maximin

We call the $y$-axis the "MM" line. Point A is above the "RD" line and to the left of the "MM" line but below the "PD" line. It lies in the region of payoff profiles that satisfy the criterion of lossdominance. For those payoff profiles, the all-0 state is uniquely stochastically stable for all degrees of loss aversion. Point B satisfies both payoff-dominance and risk-dominance. For payoff profiles in this region, the all-0 state may fail to be stochastically stable for large $\lambda$. For example, if the payoff profile is Point $\mathrm{B}$, only $a_{01}$ is regarded as a loss for an exogenous reference point $r \in\left(0, b_{x}\right]$ where $b_{x}$ is the $x$-coordinate of Point B. Then the all-0 state is not stochastically stable for $r \in\left(0, b_{x}\right]$ and all sufficiently large $\lambda$. Point $\mathrm{C}$ fails to be risk-dominant but satisfies the maximin criterion. In this region, the all-0 state is stochastically stable only for sufficiently large $\lambda$.

When strategy 0 is loss-dominant, the loss associated with $a_{10}$ is the largest loss and it has a significant negative impact on the perceived value of choosing strategy 1 for agents with higher degrees of loss-aversion. This observation implies that higher degrees of loss-aversion may help to enhance the stability of a loss-dominant equilibrium (to enlarge its basin of attraction). The next result will clarify this conjecture in terms of the size of the basin of attraction. Let $\mathcal{B}_{i}(\lambda)$ denote the basin of attraction of the all- $i$ state for the degree of loss aversion $\lambda$, that is, strategy $i$ is the unique best response for agents with $\lambda$ for all $x \in \mathcal{B}_{i}(\lambda)$. Let also

$$
L_{k}(x)=(1-x)\left(\ell_{k}\left(x, a_{00}\right)-\ell_{k}\left(x, a_{10}\right)\right)+x\left(\ell_{k}\left(x, a_{01}\right)-\ell_{k}\left(x, a_{11}\right)\right),
$$


where $\ell_{k}\left(x, a_{i j}\right)=\max \left\{r\left(s_{k}^{r}, \hat{s}_{k}, x\right)-a_{i j}, 0\right\}$ defines agent $k$ 's perceived loss of receiving $a_{i j} . L_{k}(x)$ measures the difference of the perceived losses of the two strategies for agent $k$ being in state $x$. $L_{k}(x)<0$ implies that the perceived loss associated with strategy 1 is greater than that associated with strategy 0 for agent $k . L_{k}(x)>0$ implies the opposite.

The proof of Theorem 3.2, roughly speaking, shows that $L_{k}(x)<0$ for all $x \in[0,1 / 2]$, and thus the basin of attraction of a loss-dominant equilibrium never shrinks beyond the midpoint of the state space for any degree of loss-aversion. The next corollary offers a further comparative statics property with respect to the basin of attraction. We omit the proof since it is immediate from the proof of Theorem 3.2.

Corollary 3.3. Let $x \in X$ and $\Delta u^{k}(x ; \lambda)=u_{1}\left(x, r_{k}(x ; \lambda)\right)-u_{0}\left(x, r_{k}(x ; \lambda)\right)$, where $r_{k}(x)$ denotes the reference point for agent $k$. If $L_{k}(x)<0$, then $\Delta u^{k}(x ; \lambda)$ is strictly decreasing over $\lambda$. If $L_{k}(x)>0$, then $\Delta u^{k}(x ; \lambda)$ is strictly increasing over $\lambda$. Furthermore, if $L_{k}(x)<0$ for all agents, then $x \in \mathcal{B}_{0}(\lambda)$ for all sufficiently large $\lambda$.

The function $\Delta u^{k}(x ; \lambda)$ represents the difference in reference-dependent utilities of the two strategies for agent $k$. If $\Delta u^{k}(x ; \lambda)>0$, the utility of playing strategy 1 is greater than that of playing strategy 0. Corollary 3.3 shows that if the perceived loss associated with a strategy is greater than that with the other strategy, then the utility of playing this strategy relative to the utility of playing the other strategy decreases as the degree of loss aversion increases. Therefore, a state $x$ with $L_{k}(x)<0$ must be in $\mathcal{B}_{0}(\lambda)$ for all large $\lambda$, even if $x$ is in $\mathcal{B}_{1}(\lambda)$ for small $\lambda$. Figures 2(a) and 2(b) in Section 5.1 depict $u_{1}(x)-u_{0}(x)$ in two different games, which provide an illustration for Corollary 3.3. $L_{k}(x)=0$ holds at the intersection of $u_{1}(x)-u_{0}(x)$ curves for both games. $L_{k}(x)<0$ holds for states to the left of the intersection, for which $u_{1}(x)-u_{0}(x)$ is strictly decreasing in $\lambda . L_{k}(x)>0$ holds for states to the right of the intersection, for which $u_{1}(x)-u_{0}(x)$ is strictly increasing in $\lambda$. This implies that as $\lambda$ approaches infinity, the basin of attraction of the all-0 state will converge to the $x$-coordinate of the intersection. For the game depicted in Figure 2 (a), where strategy 0 is loss-dominant, the basin of attraction of the all-0 state monotonically expands as $\lambda$ increases. For the game depicted in Figure 2 (b), where strategy 0 is not loss-dominant, the basin of attraction of the all-0 state monotonically shrinks as $\lambda$ increases.

Theorem 3.2 provides a general result that holds true for all models of reference points. We extend it to symmetric two-player normal form games in Section 4. In Section 5, we consider two specific models of reference points: social comparison and status-quo bias. For specific models of reference points, the necessary and sufficient conditions may vary. We show that a new condition 
called compensation-attractiveness plays an important role in determining stochastic stability in these models.

\section{Symmetric two-player normal form games}

In this section, we relax the assumption of two strategies and consider a class of symmetric twoplayer normal form games. The strategy space is given by $S=\{0,1,2, \ldots, K\}$. Let $X_{1 / n}=$ $\left\{0, \frac{1}{n}, \ldots, 1\right\}$. The state space for the dynamic process is given by $X=\left\{x \in X_{1 / n}^{K+1}: \sum_{i \in S} x_{i}=1\right\}$, where $x_{i}$ denotes the fraction of agents playing strategy $i$.

We extend the definitions of risk-dominance, maximin strategy and loss-dominance to symmetric two-player normal form games.

Definition 4.1. Strategy $i \in S$ is a half-dominant strategy if

$$
\alpha a_{i i}+(1-\alpha)\left(\sum_{k \neq i} \beta_{k} a_{i k}\right)>\alpha a_{j i}+(1-\alpha)\left(\sum_{k \neq i} \beta_{k} a_{j k}\right)
$$

for any $j, k \in S \backslash\{i\}, \alpha \in\left[\frac{1}{2}, 1\right], \beta_{k} \in[0,1]$, and $\sum_{k \neq i} \beta_{k}=1$.

Definition 4.2. Strategy $i \in S$ is a globally pairwise maximin strategy if

$$
\min \left\{a_{i i}, a_{i j}\right\} \geq \min \left\{a_{k i}, a_{k j}\right\}, \quad \forall j, k \in S \backslash\{i\}
$$

Definition 4.3. Strategy $i \in S$ is a generalized loss-dominant strategy if it is both a halfdominant strategy and a globally pairwise maximin strategy.

Observe that the strategy profile $(i, i)$ is a Nash equilibrium of the game if strategy $i$ is halfdominant. The condition for a globally pairwise maximin strategy is stronger than the condition that a strategy has the greatest minimum payoff, i.e., $\min _{j}\left(a_{i j}\right) \geq \min _{j}\left(a_{k j}\right)$ for all $k \neq i$. For every state, the worst possible payoff of a globally pairwise maximin strategy is at least equal to the worst possible payoff of every other strategy. We have the following result.

Theorem 4.4. Consider a symmetric two-player normal form game with $S=\{0, \ldots, K\}, K \geq 1$. The all-i state is uniquely stochastically stable for all models of reference points and all $\lambda \geq 1$ if strategy $i \in S$ is the generalized loss-dominant strategy.

We explain its intuition using an example. Suppose that the monetary payoffs are given by Table 2. Agents are loss-averse and they have reference-dependent utilities with a common reference 
Table 2: $3 \times 3$ Game (Monetary Returns)

\begin{tabular}{cc|c|c|c|} 
& \multicolumn{4}{c}{ Player 2} \\
\cline { 3 - 5 } & \multicolumn{2}{c}{0} & 1 \\
\cline { 3 - 5 } Player 1 & 0 & $a_{00}, a_{00}$ & $a_{01}, a_{10}$ & $a_{02}, a_{20}$ \\
\cline { 3 - 5 } & 1 & $a_{10}, a_{01}$ & $a_{11}, a_{11}$ & $a_{12}, a_{21}$ \\
\cline { 3 - 5 } & 2 & $a_{20}, a_{02}$ & $a_{21}, a_{12}$ & $a_{22}, a_{22}$ \\
\cline { 3 - 5 } & & \multicolumn{3}{l}{}
\end{tabular}

point $r \in \mathbb{R}$. The all-0 state being uniquely stochastically stable is implied by strategy 0 being the half-dominant strategy with respect to the reference-dependent utilities, which is equivalent to

$$
\begin{aligned}
& \frac{1}{2} v\left(a_{00}, r\right)+\frac{1}{2}\left(\beta v\left(a_{01}, r\right)+(1-\beta) v\left(a_{02}, r\right)\right) \\
& >\max \left\{\begin{array}{l}
\frac{1}{2} v\left(a_{10}, r\right)+\frac{1}{2}\left(\beta v\left(a_{11}, r\right)+(1-\beta) v\left(a_{12}, r\right)\right), \\
\frac{1}{2} v\left(a_{20}, r\right)+\frac{1}{2}\left(\beta v\left(a_{21}, r\right)+(1-\beta) v\left(a_{22}, r\right)\right)
\end{array}\right\}
\end{aligned}
$$

for any $\beta \in[0,1]$. These two conditions are equivalent to the conditions below:

$$
\begin{aligned}
& v\left(a_{00}, r\right)+v\left(a_{01}, r\right)>\max \left\{v\left(a_{10}, r\right)+v\left(a_{11}, r\right), v\left(a_{20}, r\right)+v\left(a_{21}, r\right)\right\}, \\
& v\left(a_{00}, r\right)+v\left(a_{02}, r\right)>\max \left\{v\left(a_{10}, r\right)+v\left(a_{12}, r\right), v\left(a_{20}, r\right)+v\left(a_{22}, r\right)\right\} .
\end{aligned}
$$

Theorem 3.2 suggests that $v\left(a_{00}, r\right)+v\left(a_{0 k}, r\right)>v\left(a_{j 0}, r\right)+v\left(a_{j k}, r\right)$ is implied by $a_{00}+a_{0 k}>$ $a_{j 0}+a_{j k}$ and $\min \left\{a_{00}, a_{0 k}\right\} \geq \min \left\{a_{j 0}, a_{j k}\right\}$, for $j, k \in\{1,2\}$, which are exactly captured by half-dominance on monetary payoffs (Definition 4.1) and the notion of globally pairwise maximin strategy (Definition 4.2).

The next proposition concerns two-player coordination games. A coordination game is a game with $S=\{0, \ldots, K\}$ and monetary returns $\left\{a_{i j}\right\}_{i, j \in S}$ such that $a_{i i}>a_{j i}$ for all $i, j \in S, i \neq j$. In contrast to Theorem 3.2 we have an "only-if" result for this class of games.

Proposition 4.5. Consider a two-player coordination game with $K+1$ strategies. The all-i state is uniquely stochastically stable for all models of reference points and all $\lambda \geq 1$ only if strategy $i$ is a globally pairwise maximin strategy.

Proposition 4.5 suggests that the globally pairwise maximin strategy plays a more important role for coordination games. Being the globally pairwise maximin strategy is a necessary condition for unique stochastic stability for coordination games, while it is not necessary for some normal form games. The next example illustrates the difference between the two classes of games. 
Example 3. Consider two games for which monetary returns are shown in Tables 3a and 3b. The two games differ in the value of $a_{11}$. Game $\mathrm{A}$ in Table $3 \mathrm{a}$ is a coordination game, while Game $\mathrm{B}$ in Table $3 \mathrm{~b}$ is not. For both games, strategy 0 is half-dominant and is not a globally pairwise maximin strategy. Assume that all agents adopt an exogenous reference point with $r=0$. The half-dominance of strategy 0 guarantees that the all-0 state is uniquely stochastically stable for small $\lambda$ in both games. We show that, for large $\lambda$, the all- 0 state is not stochastically stable in Game A, but it is uniquely so in Game B.

Table 3: Two $3 \times 3$ Games (Monetary returns)

(a) Game A: Coordination Game

Player 2

\begin{tabular}{|c|c|c|c|c|}
\hline & & 0 & 1 & 2 \\
\hline \multirow{3}{*}{ Player 1} & 0 & 10,10 & 1,3 & $-1,-1$ \\
\hline & 1 & 3,1 & 4,4 & $0,-2$ \\
\hline & 2 & $-1,-1$ & $-2,0$ & 1,1 \\
\hline
\end{tabular}

(b) Game B: Non-Coordination Game

Player 2

\begin{tabular}{|c|c|c|c|c|}
\hline & & 0 & 1 & 2 \\
\hline \multirow{3}{*}{ Player 1} & 0 & 10,10 & 1,3 & $-1,-1$ \\
\hline & 1 & 3,1 & 0,0 & $0,-2$ \\
\hline & 2 & $-1,-1$ & $-2,0$ & 1,1 \\
\hline
\end{tabular}

Let us first look at Game A in Table 3a. Let $\hat{x}=\left(\hat{x}_{0}, \hat{x}_{1}, \hat{x}_{2}\right)$ which satisfies $\hat{x}_{0}=\frac{n-1}{n}, \hat{x}_{1}=0$ and $\hat{x}_{2}=\frac{1}{n}$. Observe that, for sufficiently large $\lambda$,

$$
\frac{n-1}{n} \cdot 10-\frac{1}{n} \lambda<\frac{n-1}{n} \cdot 3 \Leftrightarrow u_{0}(\hat{x}, r)<u_{1}(\hat{x}, r) .
$$

Suppose that the process is in the all-0 state. The above inequality implies that one agent mutating to strategy 2 will make strategy 1 the best response for all agents and will move the process from the all-0 state to the all-1 state. By a similar argument, one mutation to strategy 1 will move the process from the all-2 state to the all-1 state. However, one mutation to either strategy 0 or 2 cannot destabilize the all-1 state. Thus, the all-1 state is uniquely stochastically stable for $r=0$ and for all sufficiently large $\lambda$.

For Game B in Table 3b, suppose that the process is in the all-0 state. Similarly to Game A, one agent mutating to strategy 2 will make strategy 1 the best response for all agents. However, the process will not move to the all- 1 state because the all- 1 state is not an equilibrium in Game $\mathrm{B}$; the best response against strategy 1 is 0 , so the process will likely move back to the all-0 state. Thus, one mutation cannot destabilize the all-0 state. On the other hand, for the all-2 state, one mutation to strategy 1 will make strategy 1 the best response, so agents will switch to strategy 1. 
Strategy 0 will eventually become the best response, and the process will move to the all-0 state. Hence, the all-0 state is uniquely stochastically stable for $r=0$ and all sufficiently large $\lambda$.

\section{Endogenous Reference Points}

In this section, we consider two models of endogenous reference points that are commonly adopted in the real life. In the first model, agents adopt the average monetary payoff in the population (social average) as their reference points. We call it the social comparison model. In the second model, agents adopt their payoffs gained from their latest games (status-quos) as their reference points. We call it the status-quo bias model.

\subsection{Social Comparison}

We consider the model in which agents' reference points are endogenously determined by social comparison. We assume that agents evaluate possible outcomes by comparing them to what people "ought to get" in the society. The average monetary payoff in the population (social average) provides a natural reference point for such agents. Let $\bar{F}(x)$ denote the average monetary payoff at state $x$, where

$$
\bar{F}(x)=(1-x)\left((1-x) a_{00}+x a_{01}\right)+x\left((1-x) a_{10}+x a_{11}\right) .
$$

\subsubsection{Prospect Dynamic with Social Comparison}

In the case of social comparison, all agents' reference points are identical and solely depend on the

population state, $r\left(s_{i}^{r}, \hat{s}_{i}, x\right)=\bar{F}(x)$. The revision probabilities can be written as a function of the population state:

$$
\begin{aligned}
& \rho_{01}(x)=\sigma^{\eta}\left(u_{1}(x, \bar{F}(x))-u_{0}(x, \bar{F}(x))\right), \\
& \rho_{10}(x)=\sigma^{\eta}\left(u_{0}(x, \bar{F}(x))-u_{1}(x, \bar{F}(x))\right),
\end{aligned}
$$

where $\sigma^{\eta}(\cdot)$ is given by Eq.(5).

For an agent playing strategy $s$, she switches to strategy $s^{\prime} \neq s$ with probability $\rho_{s s^{\prime}}(x)$. The process is a Markov chain with the state space $X$. It is characterized by the following transition 
probabilities,

$$
P_{x y}^{n}= \begin{cases}(1-x) \rho_{01}(x) & \text { if } y=x+\frac{1}{n} \\ x \rho_{10}(x) & \text { if } y=x-\frac{1}{n} \\ 1-(1-x) \rho_{01}(x)-x \rho_{10}(x) & \text { if } y=x \\ 0 & \text { otherwise. }\end{cases}
$$

We call this dynamic prospect dynamic with social comparison, which takes a familiar form of the best response dynamic with mutations because agents' reference points are only affected by the population state.

\subsubsection{Analysis}

Given the prospect dynamic with social comparison, the condition for stochastic stability of the population states for all degrees of loss-aversion is characterized as follows.

Theorem 5.1. The all-s state is uniquely stochastically stable under prospect dynamic with social comparison for all $\lambda \geq 1$ if and only if

(1) strategy $s$ is loss-dominant, or

(2) strategy $s$ is risk-dominant and $a_{s s} \geq 3\left(a_{s^{\prime} s}+a_{s^{\prime} s^{\prime}}\right)-5 a_{s s^{\prime}}$, for $s^{\prime} \neq s$.

Condition (1) of Theorem 5.1 is directly implied by Theorem 3.2. Let us focus on condition (2) of Theorem 5.1. Consider that strategy 0 is not loss-dominant but satisfies condition (2) of Theorem 5.1. This implies that $a_{00}$ is the maximum monetary return and $a_{01}$ is the minimum one. Hence, $a_{00}$ is always regarded as a gain, while $a_{00} \geq 3\left(a_{10}+a_{11}\right)-5 a_{01}$ implies that the other three payoffs are losses for $x \leq \frac{1}{2}$. In this case, the loss associated with strategy 0 is smaller than the loss associated with strategy 1 for all $x \leq \frac{1}{2}$. In other words, high $a_{00}$ compensates the potential loss incurred by choosing strategy 0 . Therefore, strategy 0 is the unique best response for $x \leq \frac{1}{2}$. We call such a strategy 0 a compensation-attractive strategy.

Intuitively, Theorem 5.1 implies that loss-averse agents prefer a strategy that is not only less risky but also unassociated with the worst outcome, unless the worst outcome is sufficiently compensated in the best case. In the following examples, we graphically illustrate the implications of Theorem 5.1.

Example 4. Consider a standard stag-hunt game, $a_{00}=2, a_{01}=2, a_{10}=0$, and $a_{11}=3$. Strategy 0 is loss-dominant. Figure 2(a) plots $u_{1}(x)-u_{0}(x)$ for $\lambda \in\{1.5,3\}$. As shown in the figure, the basin of attraction for the all-0 state is from 0 through the point at which $u_{1}(x)-u_{0}(x)=0$. 
When $\lambda=1.5$, the all-0 state has a larger basin of attraction than the all-1 state, which implies that the all-0 state is stochastically stable. When $\lambda$ increases to 3 , the all- 0 state still has a larger basin of attraction than the all-1 state. Moreover, the basin for $\lambda=3$ is larger compared to that for $\lambda=1.5$. This indicates that a loss-dominant equilibrium is more "stable" as the degree of loss-aversion increases.

Example 5. Suppose that $a_{00}=3, a_{01}=0, a_{10}=1$, and $a_{11}=1$. Strategy 0 is both riskdominant and payoff-dominant, but not loss-dominant. The game also fails to satisfy condition (2) in Theorem 5.1. Figure 2(b) plots $u_{1}(x)-u_{0}(x)$.

When $\lambda=1.5$, the all-0 state has a larger basin of attraction than the all-1 state implying that the all-0 state is stochastically stable. This indicates when the degree of loss-aversion is low, risk-dominance still plays an essential role. However, when $\lambda$ increases to 3 , the all-1 state has a larger basin of attraction than the all-0 state. Hence, the all-1 state is now uniquely stochastically stable. Given that strategy 0 is associated with the minimum payoff and fails to satisfy condition (2), strongly loss-averse agents will choose strategy 1 in the long run to avoid the loss in the worst outcome.

Example 6. Suppose that $a_{00}=7, a_{01}=1, a_{10}=2$, and $a_{11}=2$. Strategy 0 is not loss-dominant, but satisfies condition (2) of Theorem 5.1. The minimum payoff is associated with strategy 0 , but the payoff for coordinating on strategy 0 is sufficiently high. Figure $2(\mathrm{c})$ plots $u_{1}(x)-u_{0}(x)$.

Similarly to Example 4, for all degrees of loss-aversion, the all-0 state is uniquely stochastically stable. However, the basin of attraction of the all-0 state shrinks as the degree of loss-aversion increases. The minimum payoff associated with strategy 0 negatively affects the basin for the all-0 state as agents become more loss-averse.

Risk-dominance is a necessary condition for stochastic stability in Theorem 5.1 because the theorem considers all degrees of loss aversion including the loss-neutral one, $\lambda=1$. However, Theorem 5.1 also implies that when the degree of loss-aversion increases, the risk-dominance criterion becomes less important because agents care more about avoiding losses than avoiding being exposed to risks.

Next, we investigate if we can relax the risk-dominance criterion for a fixed degree of lossaversion. We find that under a certain payoff structure, a weakened criterion is enough for stochastic stability. As shown below, that criterion is associated with the concept of $p$-dominance by Morris et al. (1995). ${ }^{14}$

\footnotetext{
${ }^{14}$ See a discussion of p-dominance in sampling best response dynamic by Sandholm (2001) and Oyama et al. (2015).
} 


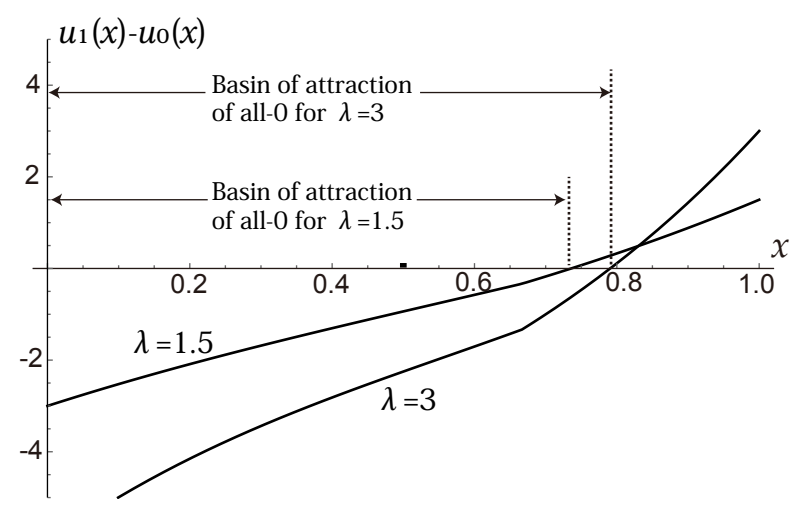

(a) $a_{00}=2, a_{01}=2, a_{10}=0, a_{11}=3$

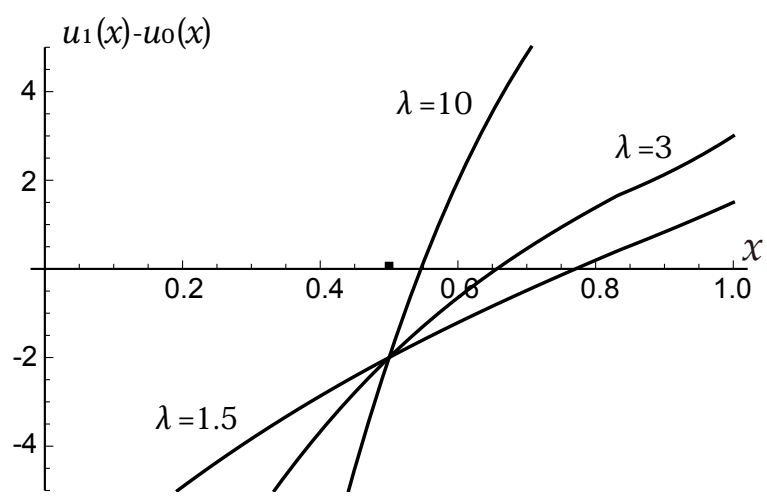

(c) $a_{00}=7, a_{01}=1, a_{10}=2, a_{11}=2$

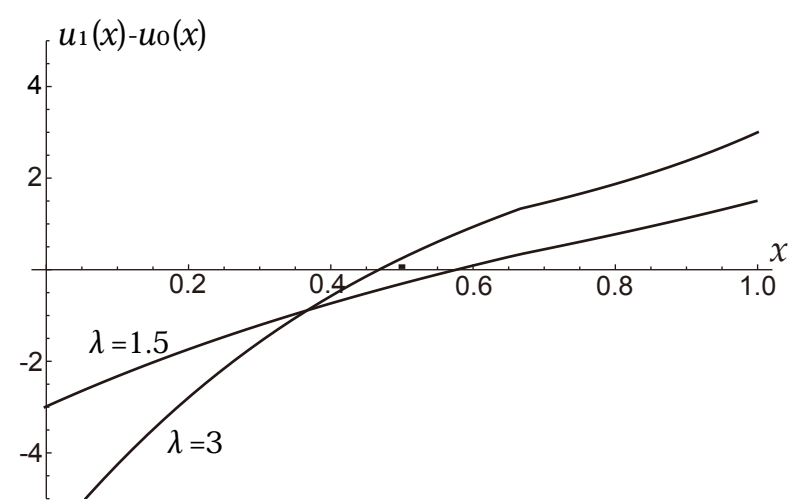

(b) $a_{00}=3, a_{01}=0, a_{10}=1, a_{11}=1$

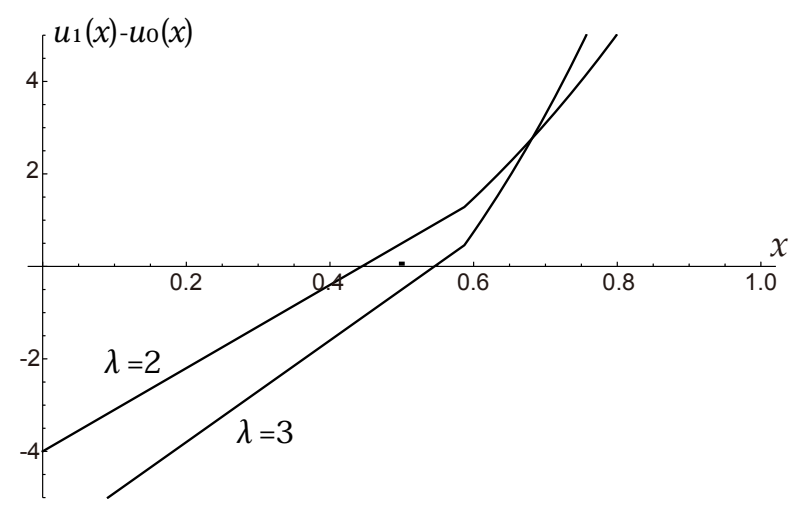

(d) $a_{00}=2, a_{01}=5, a_{10}=0, a_{11}=10$

Figure 2: $u_{1}(x)-u_{0}(x)$ for $x \in[0,1]$

Definition 5.2. Strategy $s \in\{0,1\}$ is a p-dominant strategy if for $s^{\prime} \in\{0,1\}, s^{\prime} \neq s, p a_{s s}+$ $(1-p) a_{s s^{\prime}}>p a_{s^{\prime} s}+(1-p) a_{s^{\prime} s^{\prime}}$ for $p \in[0,1]$.

Proposition 5.3. Suppose a coordination game that satisfies $\min \left\{a_{s 0}, a_{s 1}\right\}>\min \left\{a_{s^{\prime} 0}, a_{s^{\prime} 1}\right\}$ and $a_{s s^{\prime}}+a_{s^{\prime} s} \geq 2 a_{s s}$ for $s \neq s^{\prime}$. For a fixed degree of loss aversion $\lambda \geq 1$, the all-s state is uniquely stochastically stable if and only if strategy $s$ is $\frac{\lambda}{1+\lambda}$-dominant.

A strategy satisfying the conditions in Proposition 5.3 must be the maximin strategy. Note that the $\frac{\lambda}{1+\lambda}$-dominance criterion reduces to $a_{s s}>a_{s s^{\prime}}$ in the limit of large $\lambda$ and is automatically satisfied. When $\lambda$ approaches 1 , the $\frac{\lambda}{1+\lambda}$-dominance criterion reduces to risk-dominance. The next example illustrates an intuition of the proposition.

Example 7. Suppose that $a_{00}=2, a_{01}=5, a_{10}=0$, and $a_{11}=10$. Strategy 0 satisfies the payoff structure specified in Proposition 5.3. Observe that strategy 0 is $\frac{5}{7}+\varepsilon$-dominant for any $\varepsilon>0$ and 
that $\frac{\lambda}{1+\lambda}=\frac{5}{7}$ for $\lambda=2.5$. Proposition 5.3 implies that the all-0 state is uniquely stochastically stable for $\lambda>2.5$, while the all-1 state is so for $\lambda<2.5$.

Figure 2(d) plots $u_{1}(x)-u_{0}(x)$. When $\lambda=3$, the all-0 state has a larger basin of attraction than the all-1 state, which implies that the all-0 state is stochastically stable. When $\lambda=2$, however, the all-0 state has a smaller basin of attraction than the all-1 state, so it is no longer stable.

Finally, we briefly discuss how compensation-attractiveness of a strategy depends on the value of $\lambda$. Assume that strategy $s$ is risk-dominant but not the maximin strategy. For a fixed degree of loss aversion $\lambda$, the all- $s$ state is uniquely stochastically stable if $a_{s s}$ exceeds some lower bound. Such a lower bound is strictly increasing in $\lambda$. In other words, when agents are more loss-averse, they need a higher compensation for their losses. Moreover, when $\lambda$ goes to infinity, the lower bound for $a_{s s}$ converges to $3\left(a_{s^{\prime} s}+a_{s^{\prime} s^{\prime}}\right)-5 a_{s s^{\prime}}$, which is exactly the lower bound in condition (2) of Theorem 5.1.

Our result may bring some new insights to the marginal impact of payoffs on coordination in coordination games. In an experimental study on four-player weak-link games, Brandts and Cooper (2006) find that an increase in the benefits of coordination (with no change in non-coordination payoffs) leads to improved coordination, but large increases have no more impact than small increases. On the other hand, our theoretical result shows that an increase in the non-coordination payoff may have a larger impact than an increase in the coordination payoff in two-player coordination games played by loss-averse agents. As suggested by Theorem 5.1, the non-coordination payoff $a_{s s^{\prime}}$ is 5 times more important than the coordination payoff $a_{s s}$, since $a_{s s}+5 a_{s s^{\prime}}$ needs to exceed some threshold for the all- $s$ state to be stochastically stable.

\subsection{Status-Quo Bias}

Status-quo bias refers to a reference-dependent preference in which a loss-averse agent uses her current endowment as her reference point. In our setting, we assume that when an agent receives a revision opportunity, she uses the payoff she gained from her latest game as her reference point. That is, an agent's reference point $r\left(s_{i}^{r}, \hat{s}_{i}, x\right)=a_{s_{i} \hat{s}_{i}}$, where $s_{i}$ is her current strategy and $\hat{s}_{i}$ the strategy she played against in her latest match. For example, if a revising agent has gained $a_{00}$ in the latest match, her evaluations of the two strategies will be given as

$$
\begin{aligned}
& u_{0}\left(x, a_{00}\right)=x v\left(a_{01}, a_{00}\right), \\
& u_{1}\left(x, a_{00}\right)=(1-x) v\left(a_{10}, a_{00}\right)+x v\left(a_{11}, a_{00}\right) .
\end{aligned}
$$


There are potentially four different reference points that agents would use in the population: $a_{00}, a_{01}, a_{10}, a_{11}$.

\subsubsection{Prospect Dynamic with Status-Quo Bias}

Agents employ the best response with mutations (BRM) protocol as in Section 5.1. Recall that, given a revision opportunity, an agent is matched with another randomly selected agent to play the game, and then revise her strategy. Her reference point is given by the outcome of the last match. Observe that, the probability of the strategy profile being $\left(s, s^{\prime}\right)$ for the matched agents is given by the product of the proportions of agents playing the two strategies. For example, the probability that the profile of the next match will be $\left(s, s^{\prime}\right)=(0,0)$ is given by $(1-x)^{2} \cdot{ }^{15}$

The probabilities of switching depend on the reference points of the agents as follows:

$$
\begin{array}{ll}
\rho_{01}\left(x, a_{0 s}\right)=\sigma^{\eta}\left(u_{1}\left(x, a_{0 s}\right)-u_{0}\left(x, a_{0 s}\right)\right) & \text { for } s \in\{0,1\}, \\
\rho_{10}\left(x, a_{1 s}\right)=\sigma^{\eta}\left(u_{0}\left(x, a_{1 s}\right)-u_{1}\left(x, a_{1 s}\right)\right) & \text { for } s \in\{0,1\} .
\end{array}
$$

The transition probabilities of the Markov chain are given by

$$
P_{x y}^{n}= \begin{cases}(1-x)^{2} \rho_{01}\left(x, a_{00}\right)+(1-x) x \rho_{01}\left(x, a_{01}\right) & \text { if } y=x+\frac{1}{n} \\ x(1-x) \rho_{10}\left(x, a_{10}\right)+x^{2} \rho_{10}\left(x, a_{11}\right) & \text { if } y=x-\frac{1}{n} \\ 1-(1-x)^{2} \rho_{01}\left(x, a_{00}\right)-(1-x) x \rho_{01}\left(x, a_{01}\right) & \\ -x(1-x) \rho_{10}\left(x, a_{10}\right)-x^{2} \rho_{10}\left(x, a_{11}\right) & \text { if } y=x, \\ 0 & \text { otherwise. }\end{cases}
$$

We call this dynamic prospect dynamic with status-quo bias.

\subsubsection{Analysis}

We have the following result for the prospect dynamic with status-quo bias.

Theorem 5.4. The all-s state is uniquely stochastically stable under prospect dynamic with statusquo bias for any $\lambda \geq 1$ if and only if

(1) strategy $s$ is loss-dominant, or

\footnotetext{
${ }^{15}$ Note that the probability that either plays strategy 0 and the other plays strategy 1 is $2 x(1-x)$ since there are two possible cases: $\left(s, s^{\prime}\right)=(0,1)$ and $\left(s, s^{\prime}\right)=(1,0)$. Since either agent will revise, the probability that an agent with $s$ revises is given by $x(1-x)$ for each $s \in\{0,1\}$.
} 
(2) strategy $s$ is risk-dominant but not loss-dominant, and satisfies (i) $a_{s s}-a_{s^{\prime} s}>\frac{\left(a_{s^{\prime} s^{\prime}}-a_{s^{\prime}}\right)^{2}}{a_{s^{\prime} s^{\prime}}-a_{s^{\prime} s}}$ and (ii) $a_{s^{\prime} s^{\prime}}>a_{s^{\prime} s}$.

The condition (1) of Theorem 5.4 shows that loss-dominance serves as part of the necessary and sufficient condition for unique stochastic stability. This is implied by Theorem 3.2. The intuition behind the condition (2) of Theorem 5.4 can be illustrated graphically. Without loss of generality, let strategy 0 be risk-dominant but not loss-dominant. Figure 3 depicts the basins of attraction for the all-0 state and the all-1 state for sufficiently large $\lambda$.

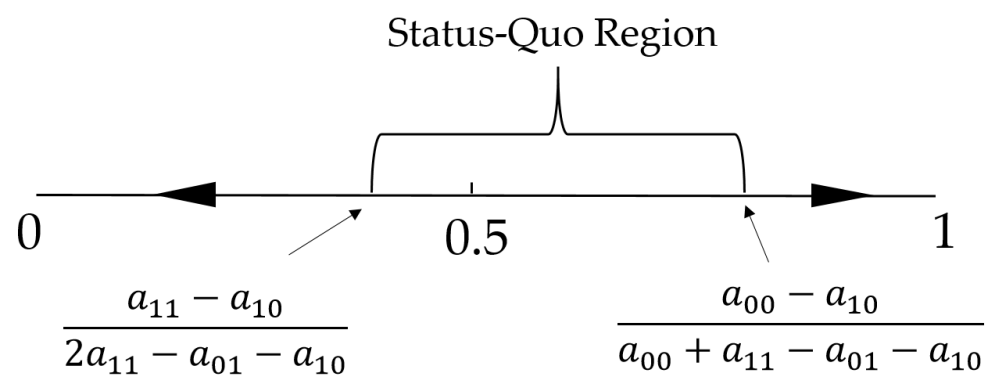

Figure 3: Basins of Attraction under Status-Quo Bias

In the status-quo region, strategy 0 is the unique best response for agents currently choosing strategy 0 , while strategy 1 is the unique best response for agents currently choosing strategy 1. Therefore, any state in this region is an absorbing state and does not contribute to determining the stochastically stable states. ${ }^{16}$ To the left of the status-quo region, for agents with reference points $a_{00}, a_{01}$ and $a_{11}$, their best response is strategy 0 ; for agents with reference point $a_{10}$, their best response is strategy 1 . This region can be viewed as the basin of attraction of the all-0 state. because no agent playing strategy 0 will switch to strategy 1 and agents playing strategy 1 will switch to strategy 0 if they are matched with agents playing strategy $1 .^{17}$

To the right of the status-quo region, strategy 1 is the unique best response for every agent when $\lambda$ is sufficiently large. Thus, the region is the basin of attraction of the all- 1 state. The condition $a_{00}-a_{10}>\frac{\left(a_{11}-a_{01}\right)^{2}}{a_{11}-a_{10}}$ in Theorem 5.4 ensures that the basin of attraction of the all-0 state is larger than that of the all-1 state. Therefore, the all-0 state is uniquely stochastically stable for all degrees of loss aversion.

\footnotetext{
${ }^{16}$ This is because the cost of moving from one basin to the other is identical to that of the opposite one.

${ }^{17}$ Since agents can be matched with themselves, any agent playing strategy 1 is matched with an agent playing strategy 1 with positive probability for all states except the all-0 state. In Section 7.2, we show that the state in which all but one agent playing strategy 0 is also stochastically stable given a matching protocol without self-matching.
} 
Note that the condition, $a_{00}-a_{10}>\frac{\left(a_{11}-a_{01}\right)^{2}}{a_{11}-a_{10}}$, is stronger than the risk-dominance, or $a_{00}-a_{10}>$ $a_{11}-a_{01}$. To see this, observe that $a_{10}>a_{01}$ implies that $a_{11}-a_{01}>a_{11}-a_{10}$. Then,

$$
a_{00}-a_{10}>\frac{\left(a_{11}-a_{01}\right)^{2}}{a_{11}-a_{10}}>a_{11}-a_{01} .
$$

It is satisfied when $a_{00}$ is sufficiently large. Similarly to the model of social comparison, when strategy 0 is compensation-attractive, the all-0 state is stochastically stable in the model of statusquo bias.

\subsection{Comparison between the two models of endogenous reference points}

In both models, when a risk-dominant strategy $s$ fails to be the maximin strategy but is compensationattractive to a certain extent, the all- $s$ state is still stochastically stable for all degrees of loss aversion. However, the mechanisms for which a strategy is sufficiently compensation-attractive are distinct across the two models. For the following discussion, assume that strategy 0 is risk-dominant but is not the maximin strategy.

For the social comparison model, as $a_{00}$ increases, the common reference point for agents, the social average, also increases. For agents with a sufficiently high degree of loss-aversion, this makes the losses associated with strategy 1 larger and makes strategy 0 more attractive. Hence, the basin of attraction of the all-0 state expands, while that for the all-1 state shrinks. Thus, the compensation induced by $a_{00}$ affects both the attractiveness of strategy 0 and that of 1 .

While, for the status-quo bias model, any change in $a_{00}$ does not affect the reference points of agents playing strategy 1. When the degree of loss-aversion is sufficiently high, the region $\left[\frac{a_{11}-a_{10}}{2 a_{11}-a_{01}-a_{10}}, 1\right]$, where agents playing strategy 1 have no incentive to switch to strategy 0 , remains unchanged as $a_{00}$ increases. That is, the attractiveness of strategy 0 is not increased for agents playing 1, and the basin of attraction of the all-0 state does not expand in response to an increase in $a_{00}$. On the other hand, an increase in $a_{00}$ makes agents playing strategy 0 more reluctant to switch to strategy 1 . Hence, the region $\left[0, \frac{a_{00}-a_{10}}{a_{00}+a_{11}-a_{01}-a_{10}}\right]$, where agents playing strategy 0 have no incentive to switch, expands toward the all-1 state. This makes the basin of attraction of the all-1 state shrink relative to that of the all-0 state.

Example 8. Similarly to Example 2, suppose a coordination game with $a_{01}=0$ and $a_{11}=1$. The parameter profiles of $\left(a_{00}, a_{10}\right)$ for which the all-0 state is uniquely stochastically stable are depicted as a gray-shaded area in Figure 4. Strategy 0 is risk-dominant for all payoff profiles above the "RD" line, i.e. $a_{00}-a_{10}>a_{11}-a_{01}=1$. The "SC" and "SQ" lines denote the necessary 
conditions for stochastic stability for the social comparison model and the status-quo bias model, respectively.

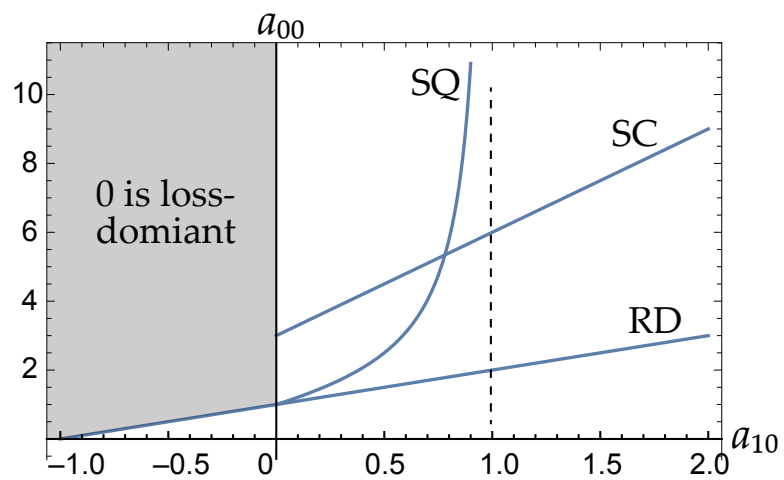

Figure 4: Regions for all-0 being stochastically stable

If $a_{00}-a_{10}>1$ and $a_{10} \leq 0$, then strategy 0 is loss-dominant and thus the all-0 state is uniquely stochastically stable for both models. For $a_{10}>0$, the regions for which the all-0 state is stochastically stable differ in the two models. For the social comparison model, if $a_{00} \geq 3 a_{10}+3$, or profiles above the "SC", the all-0 state is uniquely stochastically stable. The compensation required for the all-0 state to be stochastically stable is linear in an increase in $a_{10}$. While, for the status-quo bias model, for all profiles above the "SQ", the all-0 state is uniquely stochastically stable. As we discussed, any increase in $a_{00}$ does not raise the attractiveness of strategy 0 for agents playing 1 , which makes it increasingly difficult to compensate those agents for switching to strategy 0 in response to an increase in $a_{10}$. And it becomes impossible to attract those agents when $a_{10} \geq 1=a_{11}$, for which the all-0 state cannot be uniquely stochastically stable.

\section{Diminishing Sensitivity to Gains/Losses}

Besides loss-aversion, another important element of prospect theory is diminishing sensitivity to gains and losses. That is, people are risk-averse when dealing with gains, but are risk-seeking when dealing with losses. In this section, we investigate if loss-dominance still has predictive power when agents exhibit such diminishing sensitivity.

According to Tversky and Kahneman (1992), we modify the value function of a loss-averse agent who has a reference strategy $s_{i}^{r}$, matched with another agent playing strategy $\hat{s}_{i}$, and anticipates 
receiving the payoff $a_{s s^{\prime}}$ at the population state $x$ as follows:

$$
v\left(a_{s s^{\prime}}, r\left(s_{i}^{r}, \hat{s}_{i}, x\right)\right)= \begin{cases}\left(a_{s s^{\prime}}-r\left(s_{i}^{r}, \hat{s}_{i}, x\right)\right)^{\alpha} & \text { if } a_{s s^{\prime}}-r\left(s_{i}^{r}, \hat{s}_{i}, x\right) \geq 0, \\ -\lambda\left(r\left(s_{i}^{r}, \hat{s}_{i}, x\right)-a_{s s^{\prime}}\right)^{\alpha} & \text { otherwise }\end{cases}
$$

where $\lambda \geq 1$ and $\alpha \in(0,1]$. $\alpha$ represents the degree of diminishing sensitivity to gains and losses. ${ }^{18}$

The next theorem shows that as long as the degree of diminishing sensitivity $\alpha$ is not sufficiently small, loss-dominance still guarantees stochastic stability in any $2 \times 2$ coordination game.

Theorem 6.1. Fix a $2 \times 2$ coordination game $g$. There exists $\underline{\alpha}(g) \in(0,1)$ such that for any $\alpha \in[\underline{\alpha}(g), 1]$, the all-s state is uniquely stochastically stable for all models of reference points and all $\lambda \geq 1$ if $s$ is loss-dominant.

Theorem 6.1 generalizes Theorem 3.2 to a more general class of loss-averse preferences. It shows that for each game, there exists a lower bound for $\alpha$ that is invariant to the choice of models of reference points and the agents' degree of loss-aversion, such that loss-dominance is sufficient for stochastic stability. ${ }^{19}$

Intuitively, one should expect that when the degree of loss aversion $\lambda$ is sufficiently large, strategy $s$ being the maximin strategy still guarantees stochastic stability of the all- $s$ state because the loss associated with the worst outcome $a_{s^{\prime} s}$ is sufficiently amplified. However, if we decrease $\alpha$ while keeping $\lambda$ constant, agents will perceive less disutility in the loss frame and they become more risk-seeking. Hence, for sufficiently small $\alpha$, strategy $s$ being loss-dominant on monetary payoffs may no longer guarantee the all- $s$ state to be stochastically stable. The following example provides an illustration.

Example 9. Suppose that $a_{00}=2, a_{01}=2, a_{10}=-2.5, a_{11}=3, r=2.5$ and $\lambda=1$. Strategy 0 is loss-dominant but not payoff-dominant. When $\alpha$ is $0.4, u_{0}\left(\frac{1}{2}, r\right)-u_{1}\left(\frac{1}{2}, r\right) \approx-.2<0$, implying that the all-0 state is not stochastically stable. By a numerical computation, we can find that $\underline{\alpha}(g)$ for this game is around .49. For any $\alpha \in[.5,1]$, the all- 0 state is uniquely stochastically stable for all models of reference points and all $\lambda \geq 1$.

\footnotetext{
${ }^{18}$ Tversky and Kahneman (1992) found that people's sensitivity to gains is approximately the same as their sensitivity to losses. For simplicity, we follow their observation here. In general, an agent's sensitivity to gains can be different from her sensitivity to losses. We emphasize that allowing for different sensitivities to gains and losses would not change the main insight of this section.

${ }^{19}$ Sawa and $\mathrm{Wu}(2018)$ consider a class of preferences including loss-averse preferences with high degrees of diminishing sensitivity and show that the sufficient condition for stochastic stability must combine payoff-dominance with risk-dominance and maximin for such a class of preferences.
} 
Finally, Theorem 4.4 together with the proof of Theorem 6.1 implies that as long as the degree of diminishing sensitivity to gains and losses is not sufficiently small, generalized loss-dominance still guarantees stochastic stability in any symmetric normal form game. The proof is omitted.

Theorem 6.2. Fix a symmetric normal form game $g$ with $S=\{0, \ldots, K\}, K \geq 1$. There exists a $\underline{\alpha}(g) \in(0,1)$ such that for any $\alpha \in[\underline{\alpha}(g), 1]$, the all-i state is uniquely stochastically stable for all models of reference points and all $\lambda \geq 1$ if strategy $i \in S$ is the generalized loss-dominant strategy.

\section{Discussion}

\subsection{Heterogeneous Preferences}

In previous sections, we have shown that loss-dominance is a sufficient condition for stochastic stability for agents employing the same type of reference points with the same degree of lossaversion. In reality, agents are heterogeneous; some would be loss-neutral, other would be lossaverse, and different loss-averse agents would exhibit different degrees of the aversion and may adopt different types of reference points. Hence, it is important to consider whether our main results are robust with respect to such heterogeneous preferences. We answer the question in the affirmative; loss-dominance is still sufficient for stochastic stability.

We restate our model in order to accommodate heterogeneous agents. Our model is a dynamic process where $n$ heterogeneously loss-averse agents are (repeatedly and randomly) matched to play a coordination game where the strategy set is by $S=\{0,1\}$. The state of the process is described by their strategy profile. Let $Z \equiv S^{n}$ which denotes the state space, and $z=\left\{z_{1}, \ldots, z_{n}\right\} \in Z$ which denotes the current strategy profile, i.e., agent $k$ playing strategy $z_{k}$ for $1 \leq k \leq n$. The 'all- $i$ state' for $i \in S$ is redefined as the state being $z=\{i, \ldots, i\}$. Let $x(z)=n^{-1} \sum_{k \leq n} \mathbf{1}\left\{z_{k}=1\right\}$ which is the fraction of agents playing strategy 1, e.g., $x(z)$ corresponds to the state used in our original model. There is no essential change from the model with homogeneous agents, i.e., $n$ loss-averse agents are repeatedly matched to play a game.

The next proposition shows the robustness of Theorem 3.2. Its intuition is straightforward. Without loss of generality, let strategy 0 be loss-dominant. For any $z$ such that $x(z) \leq \frac{1}{2}$, regardless of the degrees of loss-aversion, strategy 0 is the unique best response for all agents. Hence, the basin of attraction of the all-0 state is larger than that of the all- 1 state, leading the all-0 state to be uniquely stochastically stable. 
Proposition 7.1. If $s$ is loss-dominant, then all-s state is uniquely stochastically stable in a heterogeneous population consisting of agents with any degrees of loss-aversion and any types of reference points.

Proof. Without loss of generality, assume that strategy 0 is loss-dominant. In the proof of Theorem 3.2 , we have shown that, for all $z \in Z$ such that $x(z) \in[0,1 / 2]$, strategy 0 is the unique best response for all agents with any reference point $r \in \mathbb{R}$. This implies that at least $\left\lceil\frac{n}{2}\right\rceil$ mutations are required for the process to escape from the all- 0 state. ${ }^{20}$ While, strategy 1 is not a best response for all agents for all $z \in Z$ with $x(z) \in[0,1 / 2]$. This implies that mutations less than $\left\lceil\frac{n}{2}\right\rceil$ are enough for the process to move away from the all- 1 state toward the all-0 state. Those observations imply that the all-0 state is uniquely stochastically stable.

The necessary condition for stochastic stability in a heterogeneous population depends on the detailed composition of the population. We leave it to interested readers. The next example considers agents with heterogeneous reference points. The composition of the population affects the set of stochastically stable states, and computing the set becomes more involved if there is no loss-dominant strategy.

Example 10 (heterogeneity). Consider the two coordination games shown in Table 4. Strategy 0 is loss-dominant for Game A in Table 4(a). Proposition 7.1 implies that the all-0 state is uniquely stochastically stable for any mixture of heterogeneous agents.

\section{Table 4: Two Coordination Games (Monetary Returns)}

(a) Game A: strategy 0 being loss-dominant

Player 2

\begin{tabular}{cc|c|c|} 
& \multicolumn{1}{c}{0} & \multicolumn{1}{c}{1} \\
\cline { 3 - 4 } Player 1 & 0 & 3,3 & 3,1 \\
\cline { 3 - 4 } & 1 & 1,3 & 4,4 \\
\cline { 3 - 4 } & & &
\end{tabular}

(b) Game B: no loss-dominant strategy

Player 2

\begin{tabular}{cc|c|c|}
\multicolumn{1}{c}{} & \multicolumn{1}{c}{0} & 1 \\
\cline { 3 - 4 } Player 1 & 0 & 5,5 & 1,3 \\
\cline { 3 - 4 } & 1 & 3,1 & 2,2 \\
\cline { 2 - 3 } & &
\end{tabular}

Suppose that agents play the game in Table 4(b) and have different reference points. There are two types of them; type I agents have $r_{1}=3$, while type II agents have $r_{2}=4$. Table 5 shows player 1's utilities for each type.

\footnotetext{
${ }^{20}\lceil a\rceil$ denotes the smallest integer greater than or equal to $a$.
} 
Table 5: Player 1's utilities for each type in Game B
(a) Type I: $r_{1}=3$
(b) Type II: $r_{2}=4$

\begin{tabular}{l|l|l|}
\multicolumn{1}{c}{} & \multicolumn{1}{c}{0} & \multicolumn{1}{c}{1} \\
\cline { 2 - 3 } 0 & 2 & $-2 \lambda$ \\
\cline { 2 - 3 } 1 & 0 & $-\lambda$ \\
\cline { 2 - 3 } & &
\end{tabular}

\begin{tabular}{l|l|l|} 
& \multicolumn{1}{c}{0} & \multicolumn{1}{c}{1} \\
\cline { 2 - 3 } 0 & 1 & $-3 \lambda$ \\
\cline { 2 - 3 } 1 & $-\lambda$ & $-2 \lambda$ \\
\cline { 2 - 3 } & &
\end{tabular}

For this setting, where no loss-dominant strategy exists, the stochastically stable state depends not only on $r_{1}, r_{2}$ and $\lambda$ but also on the relative proportion of each type. For example, let $\lambda=5$, $n=11$. Suppose that 2 agents are type I, and 9 agents are type II. Observe that type I agents prefer strategy 0 if and only if at least 8 agents play strategy 0 . On the other hand, type II agents become indifferent between the two strategies if 6 agents play strategy 0 and 5 agents play strategy 1. Suppose that the process is in the all-0 state. If 4 type II agents mutate to strategy 1 , then strategy 1 will become the best response for type I agents. All of type I agents will thus switch to strategy 1 , and the number of agents playing strategy 1 becomes 6 . Strategy 1 becomes a best response for type II agents and all the remaining agents playing strategy 0 may switch to strategy 1. This implies that escaping from the all-0 state requires 4 mutations. On the other hand, escaping from the all- 1 state requires at least 5 mutations since no agent will find strategy 0 a best response if $x(z)<\frac{5}{11}$. Hence, the all-1 state is uniquely stochastically stable.

In contrast to the above observation, if all agents are type II, then all agents are indifferent between the two strategies at $x(z)=\frac{5}{11}$. This implies that escaping from the all-0 state requires 6 mutations, while escaping from the all-1 state requires 5. Hence, the all-0 state is uniquely stochastically stable.

\subsection{Matching protocols without self-matching and clever revision protocols}

In this section, we consider an alternate matching protocol and an alternate decision rule, and show that our main results hold in general for different combinations of matching protocol and decision rule. The alternate matching protocol is the protocol without self-matching. When agents cannot be matched with themselves, their reference-dependent utilities of playing strategies 0 and 1 are respectively written as

$$
u_{0}\left(x, r\left(s_{i}^{r}, \hat{s}_{i}, x\right)\right)=\frac{n(1-x)-1}{n-1} v\left(a_{s 0}, r\left(s_{i}^{r}, \hat{s}_{i}, x\right)\right)+\frac{n x}{n-1} v\left(a_{s 1}, r\left(s_{i}^{r}, \hat{s}_{i}, x\right)\right),
$$




$$
u_{1}\left(x, r\left(s_{i}^{r}, \hat{s}_{i}, x\right)\right)=\frac{n(1-x)}{n-1} v\left(a_{s 0}, r\left(s_{i}^{r}, \hat{s}_{i}, x\right)\right)+\frac{n x-1}{n-1} v\left(a_{s 1}, r\left(s_{i}^{r}, \hat{s}_{i}, x\right)\right) .
$$

As for an alternate decision rule, following Kandori and Rob (1995) and Sandholm (1998), we consider a decision rule under which "clever" agents take into account the effect of their strategy change on their reference-dependent utilities. Under this criterion, the noisy best response revision protocol is given as follows.

$$
\begin{aligned}
& \rho_{01}\left(x, r_{0}\right)=\sigma^{\eta}\left(u_{1}\left(x+\frac{1}{n}, r_{0}\right)-u_{0}\left(x . r_{0}\right)\right), \\
& \rho_{10}\left(x, r_{1}\right)=\sigma^{\eta}\left(u_{0}\left(x-\frac{1}{n}, r_{1}\right)-u_{1}\left(x, r_{1}\right)\right) .
\end{aligned}
$$

Observe that the above protocol takes into account the effect of strategy change on the population state. For example, if an agent playing strategy 0 switches to strategy 1 in state $x$, then the population state will be changed to $x+\frac{1}{n} \cdot \rho_{01}\left(x, r_{0}\right)$ above takes into account such a change. $\rho_{10}\left(x, r_{0}\right)$ can be interpreted similarly. We call the revision protocol given by (2) and (3) the simple revision protocol, and the protocol given by (19) and (20) the clever revision protocol. ${ }^{21}$ We consider the following four settings:

(I) the simple revision protocol with self-matching (our primary setting),

(II) the clever revision protocol with self-matching,

(III) the simple revision protocol without self-matching,

(IV) the clever revision protocol without self-matching.

The next proposition generalizes our results to settings with clever agents and/or with the matching protocol without self-matching.

Proposition 7.2. For all sufficiently large population size $n$, Theorems 3.2 and 5.1 are valid for all (I)-(IV), while Theorem 5.4 is valid for (I),(II) and (IV).

Proof. Observe that the utilities expressed in Eq.(17) and (18) converge to the utilities expressed in Eq.(1) in the limit of the large population size, i.e.,

$$
\begin{aligned}
\lim _{n \rightarrow \infty} u_{0}\left(x, r\left(s_{i}^{r}, \hat{s}_{i}, x\right)\right) & =\lim _{n \rightarrow \infty} \frac{n(1-x)-1}{n-1} v\left(a_{s 0}, r\left(s_{i}^{r}, \hat{s}_{i}, x\right)\right)+\frac{n x}{n-1} v\left(a_{s 1}, r\left(s_{i}^{r}, \hat{s}_{i}, x\right)\right) \\
& =(1-x) v\left(a_{s 0}, r\left(s_{i}^{r}, \hat{s}_{i}, x\right)\right)+x v\left(a_{s 1}, r\left(s_{i}^{r}, \hat{s}_{i}, x\right)\right)
\end{aligned}
$$

\footnotetext{
${ }^{21}$ Rhode and Stegeman (1996) showed that the simple revision protocol together with the matching protocol without self-matching may result in selecting states which are not Nash equilibria. Using "clever" agents avoids the issue.
} 


$$
\begin{aligned}
\lim _{n \rightarrow \infty} u_{1}\left(x, r\left(s_{i}^{r}, \hat{s}_{i}, x\right)\right) & =\lim _{n \rightarrow \infty} \frac{n(1-x)}{n-1} v\left(a_{s 0}, r\left(s_{i}^{r}, \hat{s}_{i}, x\right)\right)+\frac{n x-1}{n-1} v\left(a_{s 1}, r\left(s_{i}^{r}, \hat{s}_{i}, x\right)\right) \\
& =(1-x) v\left(a_{s 0}, r\left(s_{i}^{r}, \hat{s}_{i}, x\right)\right)+x v\left(a_{s 1}, r\left(s_{i}^{r}, \hat{s}_{i}, x\right)\right) .
\end{aligned}
$$

Observe also that the utility differences, $u_{0}(\cdot)-u_{1}(\cdot)$ and $u_{1}(\cdot)-u_{0}(\cdot)$, in Eq.(19) and (20) converge to those in Eq.(2) and (3) in the large population limit, i.e.,

$$
\begin{aligned}
\lim _{n \rightarrow \infty} u_{0}\left(x-\frac{1}{n}, r\left(s_{i}^{r}, \hat{s}_{i}, x-\frac{1}{n}\right)\right) & =\lim _{n \rightarrow \infty} u_{0}\left(x, r\left(s_{i}^{r}, \hat{s}_{i}, x\right)\right) \\
& =(1-x) v\left(a_{s 0}, r\left(s_{i}^{r}, \hat{s}_{i}, x\right)\right)+x v\left(a_{s 1}, r\left(s_{i}^{r}, \hat{s}_{i}, x\right)\right), \\
\lim _{n \rightarrow \infty} u_{1}\left(x+\frac{1}{n}, r\left(s_{i}^{r}, \hat{s}_{i}, x+\frac{1}{n}\right)\right) & =\lim _{n \rightarrow \infty} u_{1}\left(x, r\left(s_{i}^{r}, \hat{s}_{i}, x\right)\right) \\
& =(1-x) v\left(a_{s 0}, r\left(s_{i}^{r}, \hat{s}_{i}, x\right)\right)+x v\left(a_{s 1}, r\left(s_{i}^{r}, \hat{s}_{i}, x\right)\right) .
\end{aligned}
$$

Hence, the limiting reference-dependent utilities in the settings (II)-(IV) coincide with the referencedependent utilities in (I), which is our main model. The claim is thus implied by the proofs of Theorems 3.2, 5.1 and 5.4.

For the setting (III) with the status-quo bias, the next corollary is implied by the proof of Theorem 5.4.

Corollary 7.3. Consider the simple revision protocol without self-matching. The all-s state and the state in which all but one agent play strategy s are both stochastically stable under prospect dynamic with status-quo bias if and only if strategy $s$ is risk-dominant but not loss-dominant, and satisfies (i) $a_{s s}-a_{s^{\prime} s}>\frac{\left(a_{s^{\prime} s^{\prime}}-a_{s s^{\prime}}\right)^{2}}{a_{s^{\prime} s^{\prime}}-a_{s^{\prime} s}}$ and (ii) $a_{s^{\prime} s^{\prime}}>a_{s^{\prime} s}$.

The intuition for Corollary 7.3 is as follows. Assume that strategy 0 satisfies the conditions (i) and (ii). The conditions imply that $a_{00}>a_{11}>a_{10}>a_{01}$. In the state in which all but one agent play strategy 0 , the only agent choosing strategy 1 must match with another agent choosing strategy 0 under the matching protocol without self-matching. Therefore, the reference point of the agent choosing strategy 1 in this state is always $a_{10}$. Given this, her best response is always strategy 1 for sufficiently large $\lambda$ because only $a_{01}$ is perceived as a loss for this agent. At the same time, the best response is strategy 0 for all other agents. Hence, this state is an absorbing state and can be stochastically stable. ${ }^{22}$

Note that if we instead consider the clever revision protocol, at the state in which all but one agent play strategy 0 , the agent playing strategy 1 can correctly anticipate that there will be no agent playing strategy 1 (thus she will not receive $a_{01}$ ) if she revises her strategy. Therefore, her best response would be strategy 0 and consequently the state is not stochastically stable.

\footnotetext{
${ }^{22}$ See also footnote 28 in the proof of Theorem 5.4.
} 


\subsection{Local Interaction Games}

Loss-dominance is a sufficient condition for a strategy to be the unique best response when at least half of the population of loss averse agents adopt the strategy. This implies that a loss-dominant strategy can be contagious in local interaction games. For simplicity, consider a circle model of Ellison (1993) in which agents are distributed around a circle and each agent is only matched with her two immediate neighbors. Let $N=\{1, \ldots, n\}$ denote the set of agents, $s_{i} \in\{0,1\}$ denote the strategy played by agent $i$, and $r_{i}$ denote the reference point of agent $i$. Let $s=\left\{s_{1}, \ldots, s_{n}\right\}$ and $s_{-i}=s \backslash\left\{s_{i}\right\}$. The reference-dependent utility of agent $i$ is given as below.

$$
u^{i}\left(s_{i}, s_{-i}, r_{i}\right)=\sum_{j \in\{i-1, i+1\}} v\left(a_{s_{i} s_{j}}, r_{i}\right) \quad \forall i \in N
$$

with a convention that $i-1=n$ for $i=1$ and $i+1=1$ for $i=n$. Assume that the revision protocol for agent $i$ is given as follows.

$$
\begin{aligned}
& \rho_{01}\left(s, r_{i}\right)=\sigma^{\eta}\left(u^{i}\left(1, s_{-i}, r_{i}\right)-u^{i}\left(0, s_{-i}, r_{i}\right)\right), \\
& \rho_{10}\left(s, r_{i}\right)=\sigma^{\eta}\left(u^{i}\left(0, s_{-i}, r_{i}\right)-u^{i}\left(1, s_{-i}, r_{i}\right)\right) .
\end{aligned}
$$

Let $s^{t}$ denote the strategy profile in period $t$. Define

$$
W(n, \varepsilon)=E\left[\min \left\{t\left|s^{t}=\{0, \ldots, 0\}\right| s^{0}=\{1, \ldots, 1\}\right],\right.
$$

where $W(n, \varepsilon)$ is the expected waiting time until all agents simultaneously play strategy 0 given that every agent starts with strategy 1 . The next proposition shows that the waiting time for a loss-dominant strategy to spread to the whole population is independent of the population size when they interact only with their neighbors. One agent switching to a loss-dominant strategy is enough for the strategy to spread. Consequently the expected waiting time is roughly given by $\varepsilon^{-1}$. We omit the proof since it is implied by Theorem 3.2 and Theorem 3 of Ellison (1993).

Proposition 7.4. Suppose that strategy 0 is loss-dominant. Consider a circle model with the initial state being the all-1 state. $W(n, \varepsilon)=O\left(\varepsilon^{-1}\right)$ for all models of reference points and all $\lambda \geq 1$.

\subsection{Synchronous learning protocols}

So far, we have considered the asynchronous learning protocol, in which exactly one agent revises in each period. We relax this assumption and consider a class of synchronous learning protocols in which a subset of agents simultaneously revise their strategies in each period. Let $N=\{1, \ldots, n\}$ 
denote the set of agents and $\mathcal{N}$ denote the set of all subsets of $N$. For each $J \in \mathcal{N}, q_{J}$ denotes the probability that exactly the agents in $J$ receive a revision opportunity. Each revising agent is matched with another randomly selected agent to play the game, and then she revises her strategy according to the revision protocol given by (2) and (3). Following Alós-Ferrer and Netzer (2015), we consider a regular revision distribution, that is, $q_{\{i\}}>0$ for all $i \in N$. We call a learning protocol with a regular revision distribution a regular synchronous learning protocol. We generalize Theorem 3.2 for all regular synchronous learning protocols.

Proposition 7.5. Consider a regular synchronous learning protocol. The all-s state is uniquely stochastically stable for all models of reference points and all $\lambda \geq 1$ if and only if $s$ is loss-dominant.

The intuition behind the proposition is that the basin of attraction of each strategy does not differ between the asynchronous and the regular synchronous learning protocols. If a strategy is loss-dominant, then its basin of attraction is greater than half of the interval $[0,1]$. This implies that the set of stochastically stable states under any regular synchronous learning protocol coincides with that under the asynchronous protocol described in Section 2.

Remark 7.6. Proposition 7.5 can also be extended to a synchronous learning protocol with $q_{N}=1$, that is, all agents simultaneously revise their strategies in each period. Again, the basin of attraction of each strategy under such a synchronous learning protocol does not differ from that under the asynchronous learning protocol. For instance, suppose that strategy 0 is loss-dominant. The basin of attraction of the all-0 state is $\left[0, x^{*}\right)$ for $x^{*}>1 / 2$. To escape from the all-0 state (under the synchronous protocol with $q_{N}=1$ ), it requires simultaneous mistakes of $n x^{*}$ agents, while simultaneous mistakes of $n\left(1-x^{*}\right)$ agents are required to escape from the all-1 state. This implies that the all-0 state is uniquely stochastically stable for sufficiently large $n$.

\subsection{Exogenous reference points}

Theorem 3.2 considers both endogenous and exogenous reference points and shows that lossdominance is a sufficient condition for unique stochastic stability for any reference point. However, when a strategy fails to be loss-dominant, whether it is stochastically stable hinges on the model of the reference point. We have examined a few compelling models of endogenous reference points in Section 5. In this section, we restrict our attention to exogenous reference points, or fixed reference points, and examine stochastically stable states. The next corollary is implied by the proof of Theorem 3.2 and it gives the exact condition for stochastic stability for exogenous reference points. 
Corollary 7.7. Suppose that $\lambda \geq 1$ and that all agents have the same exogenous reference point, $r \in \mathbb{R}$. Then, the all-s state is uniquely stochastically stable if and only if

$$
v\left(a_{s s}, r\right)+v\left(a_{s s^{\prime}}, r\right)>v\left(a_{s^{\prime} s}, r\right)+v\left(a_{s^{\prime} s^{\prime}}, r\right) \quad \text { for } s^{\prime} \neq s
$$

The next example illustrates the implication of Corollary 7.7. Observe that the game depicted in Table $4 \mathrm{~b}$ offers a case where a risk-dominant and payoff-dominant equilibrium may not be stochastically stable under the prospect dynamics.

Example 11 (dependence on the level of $r$ ). Recall the games given by Table 4 in Example 10. Suppose that all agents have the same reference point $r$. For the game in Table $4 \mathrm{a}$, strategy 0 is loss-dominant. Hence, the all-0 state is uniquely stochastically stable for all $r \in \mathbb{R}$ and all $\lambda \geq 1$.

For the game in Table 4b, strategy 0 is risk-dominant and is associated with the payoff-dominant equilibrium, while strategy 1 is the maximin strategy. For $r=0$ and $\lambda=1$, it is known that the all-0 state is uniquely stochastically stable. However, since strategy 0 is not loss-dominant, there may exist a pair of $r$ and $\lambda$ for which the all-0 state is not stochastically stable. For example, suppose $r=3$. The utilities are given by

$$
v\left(a_{00}, r\right)=2, \quad v\left(a_{01}, r\right)=-2 \lambda, \quad v\left(a_{10}, r\right)=0, \quad v\left(a_{11}, r\right)=-\lambda .
$$

Then, observe that

$$
v\left(a_{00}, r\right)+v\left(a_{01}, r\right)<v\left(a_{10}, r\right)+v\left(a_{11}, r\right) \Leftrightarrow \lambda>2 .
$$

Corollary 7.7 implies that the all-1 state is uniquely stochastically stable for $r=3$ and all $\lambda>2$.

\section{Conclusion}

This study serves an attempt to incorporate insights from behavioral economics into the study of evolutionary game theory. We propose new evolutionary dynamics called prospect dynamics, which are generated by a population of loss-averse agents being randomly matched and making repeated decisions in a $2 \times 2$ coordination game. We find that regardless of the type of reference points agents adopt, a population state in which all agents play the same strategy is stochastically stable if the strategy corresponds to a new solution concept, loss-dominance, which combines the notions of risk-dominance and maximin strategy and captures people's psychological needs to avoid not only risks but losses. We also show that this result can be extended to symmetric two-player normal 
form games by introducing a new concept, generalized loss-dominance, which combines the notions of half-dominance and globally pairwise maximin strategy, a generalization of maximin strategy. We then consider two models of endogenous reference points, the social comparison model and the status-quo bias model. We show that in both models, if a risk-dominant strategy fails to be lossdominant, the population state in which all agents play the risk-dominant strategy is stochastically stable if it is compensation-attractive, that is, the highest outcome of this strategy compensates the loss associated with it. Finally, we characterize the conditions for which our results are robust given that the agents exhibit diminishing sensitivity to gains/losses.

We hope that this study will encourage further research on how psychological biases influence the long-run behavior of individuals in society. An extension of this paper would be to consider prospect dynamics with revision protocols different from BRM, such as the logit protocol (c.f. Blume (1993)). Another line of extension would incorporate more psychological foundations into agents' decisions. For example, incorporating distributional social preferences such as inequality aversion (c.f. Fehr and Schmidt (1999) and Fehr et al. (2001)) may enable us to better understand how interdependence among individuals affect their adoption of norms. Another example would be to combine psychological game theory (c.f. Geanakoplos et al. (1989), Rabin (1993) and Battigalli and Dufwenberg (2009)) with evolutionary game theory. By doing so, we will be able to learn not only the evolution of individuals' behavior but also their beliefs and intentions.

\section{Appendix}

Proof of Theorem 3.2. We first prove the 'only-if' part and then the 'if' part. 'only-if' part:

Without loss of generality, we assume that strategy 0 is not loss-dominant and show that the all-0 state is not uniquely stochastically stable for some reference-dependent preference. If strategy 0 is not risk-dominant, then Theorem 3 of Kandori et al. (1993) implies that the all-0 state is not uniquely stochastically stable for the exogenous model with $r=0$ and $\lambda=1$. If strategy 0 is not a maximin strategy, then $a_{01}<a_{10}$ must hold. Suppose that $r$ is such that $a_{01}<r<\min \left\{a_{10}, a_{11}\right\}$. Then, the all-0 state is not stochastically stable for the exogenous model with such $r$ and sufficiently large $\lambda$. This is because such $r$ and large $\lambda$ will imply that, for all $x \in[0,1]$,

$$
\begin{aligned}
& (1-x) v\left(a_{00}, r\right)+x \lambda\left(a_{01}-r\right)<(1-x)\left(a_{10}-r\right)+x\left(a_{11}-r\right) \\
\Leftrightarrow & u_{0}(x, r)<u_{1}(x, r) .
\end{aligned}
$$


For any value of $a_{00}$, the above inequality will hold if $\lambda$ is sufficiently large. Thus, if strategy 0 is not loss-dominant, then there exist some reference-dependent models where the all-0 state is not uniquely stochastically stable.

'if' part:

Let $r \in(-\infty, \infty)$, which denotes the reference point for an agent. ${ }^{23}$ Without loss of generality, suppose that strategy 0 is the loss dominant strategy, i.e., $a_{10}=\min a_{i j}$. We examine that the all-0 state is uniquely stochastically stable for all $r$ and all $\lambda \geq 1$. For the proof, it suffices to show that $u_{0}(x, r)>u_{1}(x, r)$ for $x \in[0,1 / 2]$ and all $r \in \mathbb{R}$. This is because the strict inequality, $u_{0}(x, r)>u_{1}(x, r)$, implies that there exists some small $\delta>0$ such that $u_{0}(x, r)>u_{1}(x, r)$ for $x \in[0,1 / 2+\delta)$, which further implies that the basin of attraction of the all-0 state is strictly greater than all-1.

If $r \leq a_{10}$, then player 1's utilities are given by Table 6 .

Table 6: Player 1's utility for $r \leq a_{10}$

Table 7: Player 1's utility for $r>\max a_{i j}$

Player 2

\begin{tabular}{cc|c|c|} 
& \multicolumn{1}{c}{0} & 1 \\
\cline { 3 - 4 } Player 1 & 0 & $a_{00}-r$ & $a_{01}-r$ \\
\cline { 3 - 4 } & 1 & $a_{10}-r$ & $a_{11}-r$ \\
\cline { 3 - 4 } & & &
\end{tabular}

Player 2

\begin{tabular}{cc|cc|}
\multicolumn{1}{c}{0} & \multicolumn{1}{c}{0} \\
\cline { 3 - 3 } Player 1 & 0 & $\lambda\left(a_{00}-r\right)$ & $\lambda\left(a_{01}-r\right)$ \\
\cline { 3 - 4 } & 1 & $\lambda\left(a_{10}-r\right)$ & $\lambda\left(a_{11}-r\right)$ \\
\cline { 3 - 4 } & &
\end{tabular}

Obviously, in this case, $u_{0}(x)>u_{1}(x)$ holds for $x \in[0,1 / 2]$. Since $r$ is subtracted from each of the payoffs, $r$ does not essentially change the game. Then the risk-dominance on monetary payoffs implies that the all-0 state is uniquely stochastically stable for any $\lambda$.

Next, suppose that $r>\max _{i, j \in S} a_{i j}$. Player 1's utilities are shown in Table 7. Observe that, for $x \in[0,1 / 2]$, we have that

$$
\begin{aligned}
u_{0}(x, r)-u_{1}(x, r) & =(1-x) \lambda\left(a_{00}-r\right)+x \lambda\left(a_{01}-r\right)-(1-x) \lambda\left(a_{10}-r\right)-x \lambda\left(a_{11}-r\right) \\
& =\lambda\left((1-x) a_{00}+x a_{01}-(1-x) a_{10}-x a_{11}\right)>0 .
\end{aligned}
$$

The last inequality comes from that strategy 0 is risk dominant and $x \leq 1 / 2$. Thus, the all- 0 state is stochastically stable for any $\lambda$.

\footnotetext{
${ }^{23}$ This is an exogenous reference point. But, we will show that the loss dominant equilibrium is uniquely stochastically stable for all $r$. Then it will imply that it is sufficient for any endogenous reference point.
} 
For other cases, i.e., $\min a_{i j} \leq r \leq \max a_{i j}$, we consider three possible scenarios of the payoff structure: (i) $a_{11} \geq a_{00} \geq a_{01} \geq a_{10}$, (ii) $a_{11}>a_{01}>a_{00}>a_{10}$ and (iii) $a_{00}>a_{11}>a_{01} \geq a_{10}$. Suppose the case (i) $a_{11} \geq a_{00} \geq a_{01} \geq a_{10}$. First let $r$ be such that $a_{00} \leq r \leq a_{11}$. Player 1 's utilities are shown in Table 8 a.

Table 8: case (i) $a_{11} \geq a_{00} \geq a_{01} \geq a_{10}$
(a) $a_{00} \leq r \leq a_{11}$
(b) $a_{01} \leq r<a_{00}$
(c) $a_{10} \leq r<a_{01}$

\begin{tabular}{c|l|l|}
\multicolumn{1}{c}{0} & \multicolumn{1}{c}{1} \\
\cline { 2 - 3 } 0 & $\lambda\left(a_{00}-r\right)$ & $\lambda\left(a_{01}-r\right)$ \\
\cline { 2 - 3 } 1 & $\lambda\left(a_{10}-r\right)$ & $a_{11}-r$ \\
\cline { 2 - 3 } & &
\end{tabular}

\begin{tabular}{l|l|l|}
\multicolumn{1}{c}{0} & \multicolumn{1}{c}{1} \\
\cline { 2 - 3 } 0 & $a_{00}-r$ & $\lambda\left(a_{01}-r\right)$ \\
\cline { 2 - 3 } 1 & $\lambda\left(a_{10}-r\right)$ & $a_{11}-r$ \\
\cline { 2 - 3 } & &
\end{tabular}

\begin{tabular}{c|l|l|}
\multicolumn{1}{c}{0} & \multicolumn{1}{c}{1} \\
\cline { 2 - 3 } 0 & $a_{00}-r$ & $a_{01}-r$ \\
\cline { 2 - 3 } 1 & $\lambda\left(a_{10}-r\right)$ & $a_{11}-r$ \\
\cline { 2 - 3 } & &
\end{tabular}

Let $G(x)=(1-x) a_{00}+x a_{01}-(1-x) a_{10}-x a_{11}$. Note that $G>0$ for all $x \in[0,1 / 2]$ due to the risk dominance of strategy 0 and $a_{00}>a_{10}$. Then, observe that

$$
\begin{aligned}
u_{0}(x, r)-u_{1}(x, r) & =(1-x) \lambda\left(a_{00}-r\right)+x \lambda\left(a_{01}-r\right)-(1-x) \lambda\left(a_{10}-r\right)-x\left(a_{11}-r\right) \\
& =(\lambda-1)\left((1-x) a_{00}+x a_{01}-(1-x) a_{10}-x r\right)+G
\end{aligned}
$$

The risk dominance together with $r \leq a_{11}$ implies that the first term is strictly positive for $x \in$ $[0,1 / 2] .{ }^{24}$ Then, this with $G>0$ implies that the all-0 state is uniquely stochastically stable for any $\lambda$.

Next, Suppose that $r$ is such that $a_{01} \leq r<a_{00}$. Table 8b shows Player 1's utilities. Observe that

$$
\begin{aligned}
u_{0}(x, r)-u_{1}(x, r) & =(1-x)\left(a_{00}-r\right)+x \lambda\left(a_{01}-r\right)-(1-x) \lambda\left(a_{10}-r\right)-x\left(a_{11}-r\right) \\
& =(\lambda-1)\left((1-x)\left(r-a_{10}\right)-x\left(r-a_{01}\right)\right)+G>0 .
\end{aligned}
$$

The last strict inequality comes from that $G>0$ and that $a_{10} \leq a_{01}$, which implies that $(1-x)(r-$ $\left.a_{10}\right)-x\left(r-a_{01}\right) \geq 0$ for $x \leq 1 / 2$.

Lastly, suppose that $r$ is such that $a_{10} \leq r<a_{01}$, for which Player 1's utilities are shown in Table 8c. Observe that

$$
\begin{aligned}
u_{0}(x, r)-u_{1}(x, r) & =(1-x)\left(a_{00}-r\right)+x\left(a_{01}-r\right)-(1-x) \lambda\left(a_{10}-r\right)-x\left(a_{11}-r\right) \\
& =(\lambda-1)(1-x)\left(r-a_{10}\right)+G>0 .
\end{aligned}
$$

\footnotetext{
${ }^{24}$ To see this, observe that the first term is strictly positive even if $r=a_{11}$.
} 
$G>0$ and $r \geq a_{10}$ imply the last strict inequality. This completes the proof for the case (i).

Suppose the case (ii) $a_{11}>a_{01}>a_{00}>a_{10}$. First suppose that $r$ is such that $a_{01} \leq r \leq a_{11}$. Player 1's utilities are shown in Table 9a. This is the same as the case (i) with utilities given in Table 8a. Thus, strategy 0 is always the best response for $x \in[0,1 / 2]$.

Table 9: case (ii) $a_{11}>a_{01}>a_{00}>a_{10}$
(a) $a_{01} \leq r \leq a_{11}$
(b) $a_{00} \leq r<a_{01}$
(c) $a_{10} \leq r<a_{00}$

\begin{tabular}{c|c|c|}
\multicolumn{1}{c}{0} & \multicolumn{1}{c}{1} \\
\cline { 2 - 3 } 0 & $\lambda\left(a_{00}-r\right)$ & $\lambda\left(a_{01}-r\right)$ \\
\cline { 2 - 3 } 1 & $\lambda\left(a_{10}-r\right)$ & $a_{11}-r$ \\
\cline { 2 - 3 } & &
\end{tabular}

\begin{tabular}{c|c|c|}
\multicolumn{1}{c}{0} & \multicolumn{1}{c}{1} \\
\cline { 2 - 3 } 0 & $\lambda\left(a_{00}-r\right)$ & $a_{01}-r$ \\
\cline { 2 - 3 } 1 & $\lambda\left(a_{10}-r\right)$ & $a_{11}-r$ \\
\cline { 2 - 3 } & &
\end{tabular}

\begin{tabular}{c|l|c|}
\multicolumn{1}{c}{0} & \multicolumn{1}{c}{1} \\
\cline { 2 - 3 } 0 & $a_{00}-r$ & $a_{01}-r$ \\
\cline { 2 - 3 } 1 & $\lambda\left(a_{10}-r\right)$ & $a_{11}-r$ \\
\cline { 2 - 3 } & &
\end{tabular}

Next, suppose that $r$ is such that $a_{00} \leq r<a_{01}$, for which 1's utilities are shown in Table $9 \mathrm{~b}$. Observe that

$$
\begin{aligned}
u_{0}(x, r)-u_{1}(x, r) & =(1-x) \lambda\left(a_{00}-r\right)+x\left(a_{01}-r\right)-(1-x) \lambda\left(a_{10}-r\right)-x\left(a_{11}-r\right) \\
& =(\lambda-1)(1-x)\left(a_{00}-a_{10}\right)+G>0 .
\end{aligned}
$$

$G>0$ and $a_{00}>a_{10}$ imply the last strict inequality.

Lastly, suppose that $r$ is such that $a_{10} \leq r<a_{01}$. Player 1's utilities are shown in Table 9c. Observe that this is the same as the case (i) with Table $8 \mathrm{c}$. Thus, strategy 0 is always the best response for $x \in[0,1 / 2]$. This proves our claim for the case (ii).

Suppose the case (iii) $a_{00}>a_{11}>a_{01} \geq a_{10}$. First suppose that $r$ is such that $a_{11} \leq r \leq a_{00}$. Player 1's utilities are shown in Table 10a. Observe that

$$
\begin{aligned}
u_{0}(x, r)-u_{1}(x, r) & =(1-x)\left(a_{00}-r\right)+x \lambda\left(a_{01}-r\right)-(1-x) \lambda\left(a_{10}-r\right)-x \lambda\left(a_{11}-r\right) \\
& =(\lambda-1)\left((1-x)\left(r-a_{10}\right)-x\left(r-a_{01}\right)+x\left(r-a_{11}\right)+G>0 .\right.
\end{aligned}
$$

The last strict inequality is implied by $G>0, r \geq a_{11}$, and $a_{01} \geq a_{10}$, which implies that $(1-x)(r-$ $\left.a_{10}\right)-x\left(r-a_{01}\right) \geq 0$ for $x \in[0,1 / 2]$. Thus, strategy 0 is always the best response for $x \in[0,1 / 2]$.

Next, suppose that $r$ is such that $a_{01} \leq r<a_{11}$. Player 1's utilities are shown in Table 10b. This is the same as the case (i) with Table $8 \mathrm{~b}$. Thus, strategy 0 is always the best response for $x \in[0,1 / 2]$. Lastly, suppose that $a_{10} \leq r<a_{01}$, for which Table 10c shows 1 's utilities. This is the same as the case (i) with Table $8 \mathrm{c}$. Thus, strategy 0 is always the best response for $x \in[0,1 / 2]$.

Through the above discussion, we have shown that strategy 0 is always the best response for $x \in[0,1 / 2]$ for any $r \in \mathbb{R}$ and any $\lambda \geq 1$. This completes the proof. 
Table 10: case (iii) $a_{00}>a_{11}>a_{01} \geq a_{10}$

(a) $a_{11} \leq r \leq a_{00}$

\begin{tabular}{l|l|l|}
\multicolumn{1}{c}{} & \multicolumn{1}{c}{0} & \multicolumn{1}{c}{1} \\
\cline { 2 - 3 } 0 & $a_{00}-r$ & $\lambda\left(a_{01}-r\right)$ \\
\cline { 2 - 3 } 1 & $\lambda\left(a_{10}-r\right)$ & $\lambda\left(a_{11}-r\right)$ \\
\cline { 2 - 3 } & &
\end{tabular}

(b) $a_{01} \leq r<a_{11}$

\begin{tabular}{l|l|l|}
\multicolumn{1}{c}{} & \multicolumn{1}{c}{0} & \multicolumn{1}{c}{1} \\
\cline { 2 - 3 } 0 & $a_{00}-r$ & $\lambda\left(a_{01}-r\right)$ \\
\cline { 2 - 3 } 1 & $\lambda\left(a_{10}-r\right)$ & $a_{11}-r$ \\
\cline { 2 - 3 } & &
\end{tabular}

(c) $a_{10} \leq r<a_{01}$

\begin{tabular}{l|l|l|}
\multicolumn{1}{c}{} & \multicolumn{1}{c}{0} & \multicolumn{1}{c}{1} \\
\cline { 2 - 3 } 0 & $a_{00}-r$ & $a_{01}-r$ \\
\cline { 2 - 3 } 1 & $\lambda\left(a_{10}-r\right)$ & $a_{11}-r$ \\
\cline { 2 - 3 } & &
\end{tabular}

Proof of Theorem 4.4. Notice that if there exists a generalized loss-dominant strategy, it must be unique. Also note that the half-dominance of strategy $i$ implies that $a_{i i}>a_{j i}$ for all $j \neq i$, which further implies that,

$$
v\left(a_{i i}, r\right)>v\left(a_{j i}, r\right) \quad \forall j \in S \backslash\{i\}, r \in \mathbb{R}, \lambda \geq 1 .
$$

Inequality (7) implies that

$$
\frac{1}{2} a_{i i}+\frac{1}{2} \sum_{k \in S \backslash\{i\}} \beta_{k} a_{i k}>\frac{1}{2} a_{j i}+\frac{1}{2} \sum_{k \in S \backslash\{i\}} \beta_{k} a_{j k},
$$

for any $j \in S \backslash\{i\}, \beta_{k} \in[0,1]$, for any $k \in S \backslash\{i\}$ and $\sum_{k \in S \backslash\{i\}} \beta_{k}=1$. By letting $\beta_{k}=1$ for each $k \neq i$, inequality (29) implies that

$$
a_{i i}+a_{i k}>a_{j i}+a_{j k}, \text { for any } j, k \in S \backslash\{i\} .
$$

Observe that inequalities (8) and (30) together imply that

$$
v\left(a_{i i}, r\right)+v\left(a_{i k}, r\right)>v\left(a_{j i}, r\right)+v\left(a_{j k}, r\right), \text { for any } j, k \in S \backslash\{i\} .
$$

To see this, recall that for payoff structures with $a_{j k}>a_{i k}$, we have proved that inequality (31) holds, which has been shown in the proof of the "if" part of Theorem 3.2. ${ }^{25}$ If the payoffs are such that $a_{i k} \geq a_{j k}$, then it implies that $v\left(a_{i k}, r\right) \geq v\left(a_{j k}, r\right)$ for all $r \in \mathbb{R}$ and all $\lambda \geq 1$. This with inequality (28) implies (31).

Observe that inequalities (28) and (31) together imply that

$$
\alpha v\left(a_{i i}, r\right)+(1-\alpha)\left(\sum_{k \in S \backslash\{i\}} \beta_{k} v\left(a_{i k}, r\right)\right)>\alpha v\left(a_{j i}, r\right)+(1-\alpha)\left(\sum_{k \in S \backslash\{i\}} \beta_{k} v\left(a_{j k}, r\right)\right),
$$

for any $j \in S \backslash\{i\}, \alpha \in\left[\frac{1}{2}, 1\right], \beta_{k} \in[0,1]$, for any $k \in S \backslash\{i\}$ and $\sum_{k \in S \backslash\{i\}} \beta_{k}=1$. Hence, strategy $i$ is half-dominant with respect to the reference-dependent utility function. Corollary 1 of Ellison (2000) implies that all- $i$ state is uniquely stochastically stable.

\footnotetext{
${ }^{25}$ We have proved (31) for the case with $k=j$ in Theorem 3.2. It can be readily extended to cases with $k \neq j$.
} 
Proof of Proposition 4.5. Suppose that strategy $i$ is not a globally pairwise maximin strategy. Then there exist $j, k \neq i$ such that $\min \left\{a_{i i}, a_{i j}\right\}<\min \left\{a_{k i}, a_{k j}\right\}$. Since the game is a coordination game, $a_{i i}>a_{k i}$, which implies that $a_{i j}<\min \left\{a_{k i}, a_{k j}\right\}$. Choose $r \in \mathbb{R}$ such that $a_{i j}<r<\min \left\{a_{k i}, a_{k j}\right\}$. Let $x \in X$ be such that $x_{j}>0$ and $x_{i}=1-x_{j}$. Observe that, for any $x_{j}>0$, if $\lambda$ is sufficiently large, strategy $k$ becomes the best response: ${ }^{26}$

$$
\begin{aligned}
& (1-x) v\left(a_{i i}, r\right)+x \lambda\left(a_{i j}-r\right)<(1-x)\left(a_{k i}-r\right)+x\left(a_{k j}-r\right) \\
\Leftrightarrow & u_{i}(x, r)<u_{k}(x, r) .
\end{aligned}
$$

This implies that, for all sufficiently large $\lambda$, if an agent mutates to strategy $j$ in the all- $i$ state, then strategy $i$ will be no longer a best response. Note that, after that mutation, there is non-vanishing probability that each agent playing strategy $i$ will switch to strategy $k$ and the process will reach the all- $k$ state. This implies that one mutation to strategy $j$ can destabilize the all- $i$ state and the process will move to another equilibrium, the all- $k$ state. Then, Lemma 4 of Nöldeke and Samuelson (1993) implies that if the all- $i$ state is stochastically stable, then the all- $k$ state must be also stochastically stable. Then, the all- $i$ state cannot be uniquely so. ${ }^{27}$

Proof of Theorem 5.1. We first prove the sufficient part of the theorem. We have proved that lossdominance guarantees uniquely stochastic dominance in Theorem 3.2. So let us consider the case in which strategy 0 is a risk-dominant strategy, $\min \left\{a_{00}, a_{01}\right\}<\min \left\{a_{10}, a_{11}\right\}$ and $a_{00}+5 a_{01} \geq$ $3\left(a_{10}+a_{11}\right)$.

First we observe that $\min \left\{a_{00}, a_{01}\right\}<\min \left\{a_{10}, a_{11}\right\}$ implies $a_{01}=\min \left\{a_{00}, a_{01}, a_{10}, a_{11}\right\}$.

The risk-dominance of strategy 0 implies that $a_{00}>a_{11}$, which further implies that $\frac{2 a_{00}-a_{01}-a_{10}}{2\left(a_{00}+a_{11}-a_{10}-a_{01}\right)}>$ $\frac{1}{2}$. Therefore $\bar{F}(x)$ decreases in $x$ for $x \in\left[0, \frac{1}{2}\right]$. By the fact that $a_{01}=\min \left\{a_{00}, a_{01}, a_{10}, a_{11}\right\}$ and $a_{00}+5 a_{01} \geq 3\left(a_{10}+a_{11}\right)$, we know $\max \left\{a_{11}, a_{10}\right\}<\bar{F}\left(\frac{1}{2}\right)$. Hence, for an agent playing strategy 1 , when $x \in\left[0, \frac{1}{2}\right]$, she always regards both $a_{11}$ and $a_{10}$ as losses comparing to the reference point. The utility for an agent playing strategy 1 for $0 \leq x \leq \frac{1}{2}$ is given by

$$
u_{1}(x)=\lambda(1-x)\left(a_{10}-\bar{F}(x)\right)+\lambda x\left(a_{11}-\bar{F}(x)\right) .
$$

For an agent playing strategy 0 , she always regards $a_{00}$ as gain and $a_{01}$ as loss comparing to the reference point. Therefore, the utility for an agent playing strategy 0 for $0 \leq x \leq \frac{1}{2}$ is given by

$$
u_{0}(x)=(1-x)\left(a_{00}-\bar{F}(x)\right)+\lambda x\left(a_{01} \bar{F}(x)\right) .
$$

\footnotetext{
${ }^{26}$ Some strategy other than $k$ may become the best response. Then, let that strategy be denoted by $k$.

${ }^{27}$ Roughly speaking, Lemma 4 of Nöldeke and Samuelson (1993) says that if an equilibrium, say A, is stochastically stable and another equilibrium, say B, is one mutation away from A, then B is also stochastically stable.
} 
When $0 \leq x \leq \frac{1}{2}, u_{1}(x) \leq \lambda x\left(a_{10}-\bar{F}(x)\right)+\lambda x\left(a_{11}-\bar{F}(x)\right)$. Hence, to ensure that $u_{1}(x)-u_{0}(x)<$ 0 , for $0 \leq x \leq \frac{1}{2}$ and any $\lambda \geq 1$, it suffices to show that $\lambda x\left(a_{10}-\bar{F}(x)\right)+\lambda x\left(a_{11}-\bar{F}(x)\right)<$ $\lambda x\left(a_{01}-\bar{F}(x)\right)$ for any $0 \leq x \leq \frac{1}{2}$. When $\lambda$ is low, we use the fact that strategy 0 is risk-dominant to show that $u_{1}(x)-u_{0}(x)<0$. When $\lambda$ is high, We need $2 \bar{F}(x)-a_{11}-a_{10} \geq \bar{F}(x)-a_{01}$ for any $x \in\left(0, \frac{1}{2}\right]$, which is equivalent to $a_{00}+5 a_{01} \geq 3\left(a_{10}+a_{11}\right)$.

To conclude, we prove that if strategy 0 is a risk-dominant strategy, $\min \left\{a_{00}, a_{01}\right\}<\min \left\{a_{10}, a_{11}\right\}$ and $a_{00}+5 a_{01} \geq 3\left(a_{10}+a_{11}\right)$, then the all-0 state is uniquely stochastically stable for all $\lambda \geq 1$.

Next, we prove the necessary part of Theorem 5.1 by proving the contrapositive statement. Without loss of generality, let us focus on strategy 0 . There are two possible scenarios: 1) strategy 0 is not risk-dominant; 2) strategy 0 is risk-dominant but not a maximin strategy and $a_{00}+5 a_{01}<$ $3\left(a_{10}+a_{11}\right)$.

When strategy 0 is not risk-dominant, it is well known that the all- 0 state is not uniquely stochastically stable for $\lambda=1$. (see Kandori et al. (1993), for example.)

In the scenario in which strategy 0 is risk-dominant but not a maximin strategy and $a_{00}+5 a_{01}<$ $3\left(a_{10}+a_{11}\right)$, we fix $x \in\left(\frac{1}{2}, 1\right]$ and show that strategy 1 is the unique best response for sufficiently large $\lambda$. Then, we show that strategy 1 is the best response also for $x=\frac{1}{2}$. This will imply that the basin of attraction of strategy 1 is greater than $\frac{1}{2}$.

We already know that $a_{00} \geq a_{10}, a_{11} \geq a_{01}$ in this scenario.

There are three cases to consider, 1) $a_{11} \geq \bar{F}(x) \geq a_{10}$, 2) $a_{10} \geq \bar{F}(x) \geq a_{11}$, and 3) $\bar{F}(x)>$ $\max \left\{a_{11}, a_{10}\right\}$. For the first case, observe that

$$
u_{1}(x)-u_{0}(x)=\lambda(1-x)\left(a_{10}-\bar{F}(x)\right)+x\left(a_{11}-\bar{F}(x)\right)-(1-x)\left(a_{0}-\bar{F}(x)\right)-\lambda x\left(a_{01}-\bar{F}(x)\right) .
$$

When $\lambda \rightarrow \infty, u_{1}(x)>u_{0}(x)$, because $a_{10} \geq a_{01}$ and $x>1-x$.

Similarly, we can show that when $\lambda \rightarrow \infty, u_{1}(x)>u_{0}(x)$ in the second case.

For the third case, observe that

$u_{1}(x)-u_{0}(x)=-\lambda\left[(1-x)\left(\bar{F}(x)-a_{10}\right)+x\left(\bar{F}(x)-a_{11}\right)-x\left(\bar{F}(x)-a_{01}\right)\right]-(1-x)\left(a_{00}-\bar{F}(x)\right)$.

To show that $u_{1}(x)-u_{0}(x)>0$ for some large $\lambda$, it suffices to show that $G(x) \equiv(1-x)(\bar{F}(x)-$ $\left.a_{10}\right)+x\left(\bar{F}(x)-a_{11}\right)-x\left(\bar{F}(x)-a_{01}\right)<0$.

Observe that

$$
\begin{aligned}
G(x) & =(1-x)\left(\bar{F}(x)-a_{10}\right)+x\left(a_{01}-a_{11}\right) \\
& =(1-x)^{3} a_{00}+x\left((1-x)^{2}+1\right) a_{01}+(1-x)(x(1-x)-1) a_{10}+x(x(1-x)-1) a_{11}
\end{aligned}
$$




$$
\begin{aligned}
< & \left\{x\left(1+(1-x)^{2}\right)-5(1-x)^{3}\right\} a_{10}+\left\{3(1-x)^{3}-(1-x)(1-x(1-x))\right\} a_{10} \\
& +\left\{3(1-x)^{3}-x(1-x(1-x))\right\} a_{11}
\end{aligned}
$$

For the last inequality, we use the fact that $a_{00}+5 a_{01}<3\left(a_{10}+a_{11}\right)$. Let

$$
\begin{aligned}
& G_{1}(x) \equiv x\left(1+(1-x)^{2}\right)-5(1-x)^{3}, \\
& G_{2}(x) \equiv 3(1-x)^{3}-(1-x)(1-x(1-x)), \\
& G_{3}(x) \equiv 3(1-x)^{3}-x(1-x(1-x)) .
\end{aligned}
$$

Note that $G_{1}(x)+G_{2}(x)+G_{3}(x)=0$. Then, we have that

$$
\begin{gathered}
G(x) \leq G_{2}\left(a_{10}-a_{01}\right)+G_{3}\left(a_{11}-a_{01}\right) . \\
\frac{\partial G_{2}(x)}{\partial x}=-6 x^{2}+14 x-7,
\end{gathered}
$$

Some numerical computation reveals that $G_{2}(x), G_{3}(x)<0$ for $x \in\left(\frac{1}{2}, 1\right), G_{2}\left(\frac{1}{2}\right)=G_{2}(1)=$ $G_{3}\left(\frac{1}{2}\right)=0$, and that $G_{3}(1)<0$.

Since $a_{01} \leq a_{10}$ and $a_{01}<a_{11}$.

$$
G(x)<G_{2}\left(a_{10}-a_{01}\right)+G_{3}\left(a_{11}-a_{01}\right)<0 .
$$

$G(x)<0$ implies that when $\lambda \rightarrow \infty, u_{1}(x)-u_{0}(x)>0$ in the third case.

For $x=\frac{1}{2}$, observe that

$$
u_{1}\left(\frac{1}{2}\right)-u_{0}\left(\frac{1}{2}\right)=\frac{\lambda}{8}\left(3 a_{10}+3 a_{11}-a_{00}-5 a_{01}\right)-\frac{1}{2}\left(a_{00}-\bar{F}\left(\frac{1}{2}\right)\right) .
$$

Since $3 a_{10}+3 a_{11}-a_{00}-5 a_{01}>0$, when $\lambda \rightarrow \infty, u_{1}\left(\frac{1}{2}\right)-u_{0}\left(\frac{1}{2}\right)>0$.

The above analysis shows that there exists a $\underline{\lambda}>1$, such that for any $\lambda>\underline{\lambda}$, the basin of attraction of the all-1 state is greater than or at least $\frac{1}{2}$. Therefore, the all-0 state is not uniquely stochastically stable for some $\lambda \geq 1$.

Proof of Proposition 5.3. Without loss of generality, let strategy 0 be the strategy that satisfies the criteria in the proposition. Then the payoff structure we consider corresponds to the first scenario in case 1 in the proof of Theorem 5.1. In this scenario, when $x \in\left[0, \frac{1}{2}\right]$, for an agent playing strategy 
0, $a_{00}$ is always a loss to her and $a_{01}$ is always a gain to her. On the other hand, for an agent playing strategy $1, a_{10}$ is always a loss to her and $a_{11}$ is always a gain to her. Hence, we have

$$
\begin{aligned}
& u_{0}(x)=\lambda(1-x)\left(a_{00}-\bar{F}(x)\right)+x\left(a_{01}-\bar{F}(x)\right) \\
& u_{1}(x)=\lambda(1-x)\left(a_{10}-\bar{F}(x)\right)+x\left(a_{11}-\bar{F}(x)\right) .
\end{aligned}
$$

Therefore, $u_{0}(x)-u_{1}(x)=-x\left(a_{11}-a_{01}\right)+\lambda(1-x)\left(a_{00}-a_{10}\right)$, which is a decreasing function in $x$. Hence, to ensure that the all-0 state has a larger basin of attraction, we need $u_{0}\left(\frac{1}{2}\right)>u_{1}\left(\frac{1}{2}\right)$, which is equivalent to

$$
\frac{\lambda}{1+\lambda} a_{00}+\frac{1}{1+\lambda} a_{01}>\frac{\lambda}{1+\lambda} a_{10}+\frac{1}{1+\lambda} a_{11}
$$

which is exactly the definition of $\frac{\lambda}{1+\lambda}$-dominance.

Proof of Theorem 5.4. When strategy $s$ is loss-dominant, the stochastic stability of the all- $s$ state is implied by Theorem 3.2. Let us prove the Condition (2) of the theorem by proving the contrapositive statement. Without loss of generality, let strategy 0 be risk-dominant and satisfy $\min \left\{a_{00}, a_{01}\right\}<$ $\min \left\{a_{10}, a_{11}\right\}$. Since we must have $a_{00} \geq a_{10}$ and $a_{11} \geq a_{01}$, there are two possible scenarios: $a_{00}>a_{10} \geq a_{11} \geq a_{01}$ (with $\left.a_{10}>a_{01}\right)$, and $a_{00} \geq a_{11}>a_{10}>a_{01}$.

In the first scenario in which $a_{00}>a_{10} \geq a_{11} \geq a_{01}$ (with $a_{10}>a_{01}$ ), for an agent currently using strategy 0 and gaining the payoff of $a_{00}$, her utilities of choosing the two strategies are given by

$$
\begin{aligned}
& u_{0}\left(x, a_{00}\right)=\lambda x\left(a_{01}-a_{00}\right) \\
& u_{1}\left(x, a_{00}\right)=\lambda(1-x)\left(a_{10}-a_{00}\right)+\lambda x\left(a_{11}-a_{00}\right) .
\end{aligned}
$$

By risk-dominance, $u_{0}\left(x, a_{00}\right)>u_{1}\left(x, a_{00}\right)$ for $x \in\left[0, \frac{a_{00}-a_{10}}{a_{00}+a_{11}-a_{01}-a_{10}}\right]$.

For an agent currently using strategy 0 and gaining the payoff of $a_{01}$, her utilities of choosing the two strategies are given by

$$
\begin{aligned}
& u_{0}\left(x, a_{01}\right)=(1-x)\left(a_{00}-a_{01}\right) \\
& u_{1}\left(x, a_{01}\right)=(1-x)\left(a_{10}-a_{01}\right)+x\left(a_{11}-a_{01}\right) .
\end{aligned}
$$

By risk-dominance, $u_{0}\left(x, a_{01}\right)>u_{1}\left(x, a_{01}\right)$ for $x \in\left[0, \frac{a_{00}-a_{10}}{a_{00}+a_{11}-a_{01}-a_{10}}\right]$.

For an agent currently using strategy 1 and gaining $a_{10}$, the utilities are:

$$
u_{0}\left(x, a_{10}\right)=(1-x)\left(a_{00}-a_{10}\right)+\lambda x\left(a_{01}-a_{10}\right)
$$




$$
u_{1}\left(x, a_{10}\right)=\lambda x\left(a_{11}-a_{10}\right)
$$

When $\lambda \rightarrow \infty, u_{1}\left(x, a_{10}\right)>u_{0}\left(x, a_{10}\right)$ for any $x \in[0,1]$ because $a_{01} \leq a_{11}$.

For an agent currently using strategy 1 and gaining the payoff of $a_{11}$, her utilities of choosing the two strategies are given by

$$
\begin{aligned}
& u_{0}\left(x, a_{11}\right)=(1-x)\left(a_{00}-a_{11}\right)+\lambda x\left(a_{01}-a_{11}\right) \\
& u_{1}\left(x, a_{11}\right)=(1-x)\left(a_{10}-a_{11}\right) .
\end{aligned}
$$

When $\lambda \rightarrow \infty, u_{1}\left(x, a_{11}\right)>u_{0}\left(x, a_{11}\right)$ for any $x \in[0,1]$.

Therefore, when $\lambda \rightarrow \infty$, each state in $\left[0,\left(a_{00}-a_{10}\right) /\left(a_{00}+a_{11}-a_{01}-a_{10}\right)\right)$ is an absorbing state and $\left(\frac{a_{00}-a_{10}}{a_{00}+a_{11}-a_{01}-a_{10}}, 1\right]$ is the basin of attraction of the all- 1 state. Hence, the all- 1 state is uniquely stochastically stable. This implies that the all-0 state cannot be stochastically stable if $a_{10} \geq a_{11}$.

Next, let us consider the second scenario in which $a_{00} \geq a_{11}>a_{10}>a_{01}$. Similarly to the first scenario, for agents using strategy 0 , their best response is strategy 0 for $x \in\left[0, \frac{a_{00}-a_{10}}{a_{00}+a_{11}-a_{01}-a_{10}}\right]$, given any degree of loss aversion.

For an agent currently using strategy 1 and gaining the payoff of $a_{10}$, her utilities of choosing the two strategies are given by

$$
\begin{aligned}
& u_{0}\left(x, a_{10}\right)=(1-x)\left(a_{00}-a_{10}\right)+\lambda x\left(a_{01}-a_{10}\right) \\
& u_{1}\left(x, a_{10}\right)=x\left(a_{11}-a_{10}\right) .
\end{aligned}
$$

When $\lambda \rightarrow \infty, u_{1}\left(x, a_{10}\right)>u_{0}\left(x, a_{10}\right)$ for any $x \in[0,1]$

For an agent currently using strategy 1 and gaining the payoff of $a_{11}$, her utilities of choosing the two strategies are given by

$$
\begin{aligned}
& u_{0}\left(x, a_{11}\right)=(1-x)\left(a_{00}-a_{11}\right)+\lambda x\left(a_{01}-a_{11}\right) \\
& u_{1}\left(x, a_{11}\right)=\lambda(1-x)\left(a_{10}-a_{11}\right) .
\end{aligned}
$$

When $\lambda \rightarrow \infty$, we have $u_{1}\left(x, a_{11}\right)>u_{0}\left(x, a_{11}\right)$ for $x \in\left(\frac{a_{11}-a_{10}}{2 a_{11}-a_{01}-a_{10}}, 1\right]$.

To summarize, when $\lambda \rightarrow \infty$, in the region of $\left[0, \frac{a_{11}-a_{10}}{2 a_{11}-a_{01}-a_{10}}\right)$, strategy 0 is the best response for agents currently choosing strategy 0 and agents currently choosing strategy 1 and gaining $a_{11}$. On the other hand, strategy 1 is the best response for agents currently choosing strategy 1 and gaining $a_{10}$. However, for any agent choosing strategy 1 , there is positive probability that she is 
matched with herself and $a_{11}$ becomes her reference point. Thus, the region of $\left[0, \frac{a_{11}-a_{10}}{2 a_{11}-a_{01}-a_{10}}\right)$ is the basin of attraction of strategy $0 .^{28}$

In the region of $\left(\frac{a_{00}-a_{10}}{a_{00}+a_{11}-a_{01}-a_{10}}, 1\right]$, strategy 1 is the best response for all agents.

In the region of $\left(\frac{a_{11}-a_{10}}{2 a_{11}-a_{01}-a_{10}}, \frac{a_{00}-a_{10}}{a_{00}+a_{11}-a_{01}-a_{10}}\right)$, the unique best response for agents currently playing strategy 0 is still strategy 0 while the unique best response for agents currently playing strategy 1 is still strategy 1 . Hence, each state in this region is an absorbing state.

Therefore, the size of the basin of attraction for the all- 0 state is $\frac{a_{11}-a_{10}}{2 a_{11}-a_{01}-a_{10}}$ and the size of the basin of attraction for the all- 1 state is $1-\frac{a_{00}-a_{10}}{a_{00}+a_{11}-a_{01}-a_{10}}$. When $\frac{a_{11}-a_{10}}{2 a_{11}-a_{01}-a_{10}}>1-\frac{a_{00}-a_{10}}{a_{00}+a_{11}-a_{01}-a_{10}}$, we have $a_{00}-a_{10}>\frac{\left(a_{11}-a_{01}\right)^{2}}{a_{11}-a_{10}}$. Hence, when the inequality holds, the all-0 state is uniquely stochastically stable. When $\frac{a_{11}-a_{10}}{2 a_{11}-a_{01}-a_{10}} \leq 1-\frac{a_{00}-a_{10}}{a_{00}+a_{11}-a_{01}-a_{10}}$, the all-0 state is not uniquely stochastically stable.

To conclude, in the second scenario $\left(a_{11}>a_{10}\right)$, the all-0 state is uniquely stochastically stable if and only if the condition $a_{00}-a_{10}>\frac{\left(a_{11}-a_{01}\right)^{2}}{a_{11}-a_{10}}$ holds.

Lastly, when strategy 0 is not risk-dominant, for $\lambda$ sufficiently close to 1 , the all-0 state cannot be uniquely stochastically stable.

Proof of Theorem 6.1. Without loss of generality, assume that strategy 0 is loss-dominant. For notational convenience, let $h(x)=x^{\alpha}$. Note that given a reference point $r, u_{1}(x, r)-u_{0}(x, r)$ is a linear function of $x . u_{0}(0, r)-u_{1}(0, r)>0$ is guaranteed by $a_{00}>a_{10}$, so as long as we show that $u_{0}\left(\frac{1}{2}, r\right)-u_{1}\left(\frac{1}{2}, r\right)>0$, we prove that strategy 0 is uniquely stochastically stable.

Case 1: $a_{11} \geq a_{00} \geq a_{01}>a_{10}$.

1) Suppose $r \leq a_{10}$. In this case $u_{0}\left(\frac{1}{2}, r\right)-u_{1}\left(\frac{1}{2}, r\right)=\frac{1}{2}\left(h\left(a_{00}-r\right)+h\left(a_{01}-r\right)-h\left(a_{10}-r\right)-h\left(a_{11}-r\right)\right)$.

Consider two lotteries: $L$ that gives $a_{00}-r$ with probability $\frac{1}{2}$ and gives $a_{01}-r$ with probability $\frac{1}{2}$; and $L^{\prime}$ that gives $a_{10}-r$ with probability $\frac{1}{2}$ and gives $a_{11}-r$ with probability $\frac{1}{2}$. We want to show that $L$ is strictly preferred to $L^{\prime}$ for any risk-averse decision maker. To prove this, construct a third lottery $L^{\prime \prime}$ that gives $a_{10}-r$ with probability $\frac{a_{11}-\frac{1}{2}\left(a_{00}+a_{01}\right)}{a_{11}-a_{10}}$ and gives $a_{11}-r$ with probability $\frac{\frac{1}{2}\left(a_{00}+a_{01}\right)-a_{10}}{a_{11}-a_{10}}$.

Note that $L$ and $L^{\prime \prime}$ have the same mean, i.e., $E[L]=E\left[L^{\prime \prime}\right]$. we first show that $L$ secondorder stochastically dominates $L^{\prime \prime}$. Let $F_{L}$ denote the cdf associated with $L$ and $F_{L^{\prime \prime}}$ denote the

\footnotetext{
${ }^{28}$ For the matching protocol without self-matching, when there is exactly one agent choosing strategy 1 , she always matches with another agent choosing strategy 0 . Therefore, the state in which all but 1 agents play strategy 0 is a candidate for a stochastically stable state for the matching protocol without self-matching.
} 
cdf associated with $L$. For any $y \in\left(\infty, a_{10}-r\right), \int_{\infty}^{y} F_{L}(y) d y=\int_{\infty}^{y} F_{L^{\prime \prime}}(y) d y=0$. For any $y \in\left[a_{10}-r, a_{01}-r\right), \int_{\infty}^{y} F_{L}(y) d y=0 \leq \int_{\infty}^{y} F_{L^{\prime \prime}}(y) d y=\frac{a_{11}-\frac{1}{2}\left(a_{00}+a_{01}\right)}{a_{11}-a_{10}}\left(y-\left(a_{10}-r\right)\right)$. For any $y \in$ $\left[a_{01}-r, a_{00}-r\right)$, let $m(y)=\int_{\infty}^{y} F_{L^{\prime \prime}}(y) d y-\int_{\infty}^{y} F_{L}(y) d y=\frac{a_{11}-\frac{1}{2}\left(a_{00}+a_{01}\right)}{a_{11}-a_{10}}\left(y-\left(a_{10}-r\right)\right)-\frac{1}{2}\left(y-\left(a_{01}-r\right)\right)$, which is a linear function in $y$. Since, $m\left(a_{10}-r\right)>0$ and $m\left(a_{00}-r\right)=\frac{a_{11}-a_{00}}{2\left(a_{11}-a_{10}\right)}\left(a_{00}+a_{01}-2 a_{10}\right) \geq 0$, we conclude that $m(y) \geq 0$, for any $y \in\left[a_{01}-r, a_{00}-r\right)$. For any $y \in\left[a_{00}-r, a_{11}-r\right]$, let $n(y)=\int_{\infty}^{y} F_{L^{\prime \prime}}(y) d y-\int_{\infty}^{y} F_{L}(y) d y=\frac{a_{11}-\frac{1}{2}\left(a_{00}+a_{01}\right)}{a_{11}-a_{10}}\left(y-\left(a_{10}-r\right)\right)-\frac{1}{2}\left(a_{00}-a_{01}\right)-\left(y-a_{00}\right)$, which is a linear function in $y$. Since, $n\left(a_{00}-r\right)>0$ and $n\left(a_{11}-r\right)=0$, we conclude that $n(y) \geq 0$, for any $y \in\left[a_{00}-r, a_{11}-r\right]$. When $y>a_{11}$, we have $\int_{\infty}^{y} F_{L}(y) d y=\int_{\infty}^{y} F_{L^{\prime \prime}}(y) d y$. In sum, we prove that $\int_{\infty}^{y} F_{L}(y) d y \leq \int_{\infty}^{y} F_{L^{\prime \prime}}(y) d y$ for any $y \in R$, implying that $L$ second-order stochastically dominates $L^{\prime \prime}$.

In addition, $L^{\prime \prime}$ first-order stochastically dominates $L^{\prime}$ because strategy 0 being risk-dominant implies that $\frac{a_{11}-\frac{1}{2}\left(a_{00}+a_{01}\right)}{a_{11}-a_{10}}<\frac{\frac{1}{2}\left(a_{00}+a_{01}\right)-a_{10}}{a_{11}-a_{10}}$. Hence, we conclude that any risk-averse decision maker strictly prefers $L$ to $L^{\prime}$, implying that $u_{0}\left(\frac{1}{2}, r\right)-u_{1}\left(\frac{1}{2}, r\right)>0$.

2) Suppose $r \in\left[a_{10}, a_{01}\right]$. In this case, $u_{0}\left(\frac{1}{2}, r\right)-u_{1}\left(\frac{1}{2}, r\right)=\frac{1}{2}\left(h\left(a_{00}-r\right)+h\left(a_{01}-r\right)+\lambda h(r-\right.$ $\left.\left.a_{10}\right)-h\left(a_{11}-r\right)\right)$, which is a linear function of $\lambda$. When $\lambda \rightarrow \infty, u_{0}\left(\frac{1}{2}, r\right)-u_{1}\left(\frac{1}{2}, r\right)>0$ regardless of the value of $\alpha$. When $\lambda=1$, define $f(r)=2\left(u_{0}\left(\frac{1}{2}, r\right)-u_{1}\left(\frac{1}{2}, r\right)\right)$. We have $f^{\prime \prime}(r)=h^{\prime \prime}\left(a_{00}-r\right)+h^{\prime \prime}\left(a_{01}-r\right)+h^{\prime \prime}\left(r-a_{10}\right)-h^{\prime \prime}\left(a_{11}-r\right)$. When $\alpha<1$, we have $h^{\prime \prime \prime}>0$, implying that $h^{\prime \prime}\left(a_{00}-r\right)-h^{\prime \prime}\left(a_{11}-r\right)<0$, together with $h^{\prime \prime}<0$, we have $f^{\prime \prime}(r)<0$. Hence, $f(r)$ takes its minimum either at $a_{10}$ or at $a_{01}$ for any $\alpha \in(0,1)$. We already know that when $\alpha=1, f\left(a_{10}\right)>0$ and $f\left(a_{01}\right)>0$. By continuity of $f$, there exists a $\underline{\alpha}_{1} \in(0,1)$ such that for any $\alpha \in\left[\underline{\alpha}_{1}, 1\right], f\left(a_{10}\right)>0$ and $f\left(a_{01}\right)>0$, implying that $f(r)>0$, for any $r \in\left[a_{10}, a_{01}\right]$.

3) Suppose $r \in\left(a_{01}, a_{00}\right]$. In this case, $u_{0}\left(\frac{1}{2}, r\right)-u_{1}\left(\frac{1}{2}, r\right)=\frac{1}{2}\left(h\left(a_{00}-r\right)-\lambda h\left(r-a_{01}\right)+\lambda h(r-\right.$ $\left.\left.a_{10}\right)-h\left(a_{11}-r\right)\right)$, which is a linear function of $\lambda$. When $\lambda \rightarrow \infty, u_{0}\left(\frac{1}{2}, r\right)-u_{1}\left(\frac{1}{2}, r\right)>0$ regardless of the value of $\alpha$ because $a_{01}>a_{10}$. When $\lambda=1$, define $f(r)=2\left(u_{0}\left(\frac{1}{2}, r\right)-u_{1}\left(\frac{1}{2}, r\right)\right)$. We have $f^{\prime}(r)=-h^{\prime}\left(a_{00}-r\right)-h^{\prime}\left(r-a_{01}\right)+h^{\prime}\left(r-a_{10}\right)+h^{\prime}\left(a_{11}-r\right)$. When $\alpha<1$, we have $h^{\prime \prime}<0$, implying that $-h^{\prime}\left(a_{00}-r\right)+h^{\prime}\left(a_{11}-r\right)<0$ and $-h^{\prime}\left(r-a_{01}\right)+h^{\prime}\left(r-a_{10}\right)<0$. Hence, $f^{\prime}(r)<0$ and $f(r)$ takes its minimum at $a_{00}$ for any $\alpha \in(0,1)$. We already know that when $\alpha=1, f\left(a_{00}\right)>0$. By continuity of $f$, there exist $\underline{\alpha}_{2} \in(0,1)$ such that for any $\alpha \in\left[\underline{\alpha}_{2}, 1\right], f\left(a_{00}\right)>0$, implying that $f(r)>0$, for any $r \in\left[a_{01}, a_{00}\right]$.

4) Suppose $r \in\left(a_{00}, a_{11}\right)$. In this case, $u_{0}\left(\frac{1}{2}, r\right)-u_{1}\left(\frac{1}{2}, r\right)=\frac{1}{2}\left(-\lambda h\left(r-a_{00}\right)-\lambda h\left(r-a_{01}\right)+\lambda h(r-\right.$ 
$\left.\left.a_{10}\right)-h\left(a_{11}-r\right)\right)$, which is a linear function of $\lambda$. For $\lambda=1$ and any given $\alpha$, if $u_{0}\left(\frac{1}{2}, r\right)-u_{1}\left(\frac{1}{2}, r\right)>0$, then $-h\left(r-a_{00}\right)-h\left(r-a_{01}\right)+h\left(r-a_{10}\right)>0$, which implies that $u_{0}\left(\frac{1}{2}, r\right)-u_{1}\left(\frac{1}{2}, r\right)>0$ for all $\lambda \geq 1$. Hence, it suffices to consider the case of $\lambda=1$.

Define $f(r)=2\left(u_{0}\left(\frac{1}{2}, r\right)-u_{1}\left(\frac{1}{2}, r\right)\right)$. We have $f^{\prime}(r)=-h^{\prime}\left(r-a_{00}\right)-h^{\prime}\left(r-a_{01}\right)+h^{\prime}(r-$ $\left.a_{10}\right)+h^{\prime}\left(a_{11}-r\right)$. For $\alpha<0, \lim _{r \searrow a_{00}} f^{\prime}(r)=-\infty, \lim _{r} \nearrow a_{11} f^{\prime}(r)=\infty$. We also have $f^{\prime \prime}(r)=$ $-h^{\prime \prime}\left(r-a_{00}\right)-h^{\prime \prime}\left(r-a_{01}\right)+h^{\prime \prime}\left(r-a_{10}\right)-h^{\prime \prime}\left(a_{11}-r\right)$. When $\alpha<1$, we have $h^{\prime \prime \prime}>0$, implying that $-h^{\prime \prime}\left(r-a_{01}\right)+h^{\prime \prime}\left(r-a_{10}\right)>0$, together with $h^{\prime \prime}<0$, we have $f^{\prime \prime}(r)>0$. Hence, for any $\alpha \in(0,1)$, there exists a unique solution to $\min _{r \in\left(a_{00}, a_{11}\right)} f(r)$, denoted as $r^{*}(\alpha)$, which satisfies $f^{\prime}\left(r^{*}(\alpha)\right)=0 . .^{29}$

Define $\tilde{f}(\alpha)=f\left(r^{*}(\alpha)\right)$, which is a continuous function in $\alpha$ on $(0,1)$ as well. By the fact that strategy 0 is risk-dominant, when $\alpha=1, f(r)>0$ for any $r \in\left(a_{00}, a_{11}\right]$. Hence, by extending the domain of $\tilde{f}$ to $(0,1]$, we have $\lim _{\alpha \rightarrow 1} \tilde{f}(\alpha)>0$. By continuity of $\tilde{f}$, there exist $\underline{\alpha}_{3} \in(0,1)$ such that for any $\alpha \in\left[\underline{\alpha}_{3}, 1\right], \tilde{f}(\alpha)>0$, implying that $f(r)>0$, for any $r \in\left[a_{01}, a_{00}\right]$.

5) Suppose $r \geq a_{11}$. In this case $u_{0}\left(\frac{1}{2}, r\right)-u_{1}\left(\frac{1}{2}, r\right)=\frac{\lambda}{2}\left(-h\left(r-a_{00}\right)-h\left(r-a_{01}\right)+h\left(r-a_{10}\right)+h(r-\right.$ $\left.\left.a_{11}\right)\right)$. We first prove that if $u_{0}\left(\frac{1}{2}, r\right)-u_{1}\left(\frac{1}{2}, r\right)>0$ for $r=a_{11}$, then $u_{0}\left(\frac{1}{2}, r\right)-u_{1}\left(\frac{1}{2}, r\right)>0$ for all sufficiently large $r$. Let $f(r)=-h\left(r-a_{00}\right)-h\left(r-a_{01}\right)+h\left(r-a_{10}\right)+h\left(r-a_{11}\right)$. Let $d_{00}=a_{11}-a_{00}$, $d_{01}=a_{11}-a_{01}$ and $d_{10}=a_{11}-a_{10}$. Risk-dominance of strategy 0 implies that $d_{10}>d_{00}+d_{01}$. Choose $\underline{\alpha}_{4}<1$ and $\delta>0$ such that $d_{10}^{\alpha} \geq d_{00}^{\alpha}+d_{01}^{\alpha}+\delta$ for all $\alpha \geq \underline{\alpha}_{4}$. Let $d=r-a_{11}$. Observe that for $\alpha \in\left[\underline{\alpha}_{4}, 1\right)$,

$$
\begin{aligned}
f(r) & =\left(d_{10}+d\right)^{\alpha}+d^{\alpha}-\left(d_{00}+d\right)^{\alpha}-\left(d_{01}+d\right)^{\alpha} \\
& \geq\left(\left(d_{00}^{\alpha}+d_{01}^{\alpha}+\delta\right)^{1 / \alpha}+d\right)^{\alpha}+d^{\alpha}-\left(d_{00}+d\right)^{\alpha}-\left(d_{01}+d\right)^{\alpha} \\
& >\left(d_{00}+d+\left(d_{01}^{\alpha}+\delta\right)^{1 / \alpha}\right)^{\alpha}+d^{\alpha}-\left(d_{00}+d\right)^{\alpha}-\left(d_{01}+d\right)^{\alpha} .
\end{aligned}
$$

For the last inequality, note that $x^{1 / \alpha}$ is a convex function if $\alpha<1$. Let $D_{01}=\left(d_{01}^{\alpha}+\delta\right)^{1 / \alpha}$. Note that $D_{01}>d_{01}$ for all $\alpha \leq 1$. The mean value theorem implies that $(a+b)^{\alpha} \geq a^{\alpha}+b \alpha(a+b)^{\alpha-1}$ for any $0<\alpha<1$. Applying the mean value theorem to the above inequality, we obtain

$$
\begin{aligned}
f(r) & >\left(d_{00}+d\right)^{\alpha}+\alpha D_{01}\left(d_{00}+D_{01}+d\right)^{\alpha-1}+d^{\alpha}-\left(d_{00}+d\right)^{\alpha}-\left(d_{01}+d\right)^{\alpha} \\
& =\alpha D_{01}\left(d_{00}+D_{01}+d\right)^{\alpha-1}-\left(\left(d_{01}+d\right)^{\alpha}-d^{\alpha}\right) .
\end{aligned}
$$

\footnotetext{
${ }^{29} r^{*}(\alpha)$ is a $C^{1}$ function. Fix $\alpha_{1} \in(0,1)$. Since $f^{\prime \prime} \neq 0$, the implicit function theorem implies that there is a $C^{1}$ function $\tilde{r}(\alpha)$ such that $f^{\prime}(\tilde{r}(\alpha))=0$ for all $\alpha$ in some neighborhood $L$ of $\alpha_{1}$. Since $r^{*}(\alpha)$ is unique and $f^{\prime}\left(r^{*}(\alpha)\right)=0$ must hold for all $\alpha \in L$, it must be that $r^{*}(\alpha)=\tilde{r}(\alpha)$ on $L$.
} 
Observe that

$$
\begin{aligned}
& \lim _{d \rightarrow \infty} \frac{\alpha D_{01}\left(d_{00}+D_{01}+d\right)^{\alpha-1}}{\left(d_{01}+d\right)^{\alpha}-d^{\alpha}}=\lim _{d \rightarrow \infty} \frac{\alpha D_{01}\left(d_{00}+D_{01}+d\right)^{\alpha-1} d^{-\alpha}}{\left(\frac{d_{01}+d}{d}\right)^{\alpha}-1} \\
= & \lim _{d \rightarrow \infty} \frac{\alpha D_{01}\left[(\alpha-1)\left(d_{00}+D_{01}+d\right)^{\alpha-2} d^{-\alpha}-\alpha\left(d_{00}+D_{01}+d\right)^{\alpha-1} d^{-\alpha-1}\right]}{-\alpha\left(\frac{d_{01}+d}{d}\right)^{\alpha-1} \cdot \frac{d_{01}}{d^{2}}} \\
= & \lim _{d \rightarrow \infty} \frac{\alpha D_{01}\left[(\alpha-1)\left(d_{00}+D_{01}+d\right)^{\alpha-2} d^{2-\alpha}-\alpha\left(d_{00}+D_{01}+d\right)^{\alpha-1} d^{1-\alpha}\right]}{-\alpha\left(\frac{d_{01}+d}{d}\right)^{\alpha-1} d_{01}} \\
= & \frac{D_{01}}{d_{01}}>1 .
\end{aligned}
$$

We apply the L'Hôpital's rule in the second equality. For the last equality, observe that $\lim _{d \rightarrow \infty}\left(d_{00}+\right.$ $\left.D_{01}+d\right)^{\alpha-2} d^{2-\alpha}=1, \lim _{d \rightarrow \infty}\left(d_{00}+D_{01}+d\right)^{\alpha-1} d^{1-\alpha}=1$ and $\lim _{d \rightarrow \infty}\left(\frac{d_{01}+d}{d}\right)^{\alpha-1}=1$. This observation implies that there exists a large $M>0$ such that $f(r)>0$ for all $r>M$.

Next we consider $r \leq M . h^{\prime}(\cdot)>0$ and $h^{\prime \prime}(\cdot)<0$ imply that $h\left(r-a_{11}\right)-h\left(r-a_{00}\right)$ is strictly increasing in $r$ and $h\left(r-a_{10}\right)-h\left(r-a_{01}\right)$ is strictly decreasing in $r$. Hence, we have $f(r)>h\left(r-a_{10}\right)-h\left(r-a_{01}\right)-h\left(a_{11}-a_{00}\right)$. When $\alpha=1, h\left(r-a_{10}\right)-h\left(r-a_{01}\right)-h\left(a_{11}-a_{00}\right)>0$ by the fact that strategy 0 is risk-dominant. By continuity of $h(\cdot)$ on $\alpha$, there exists $\underline{\alpha}_{5} \in(0,1)$, such that for any $\alpha \in\left[\underline{\alpha}_{5}, 1\right], f(r)>0$ for all $r \leq M$.

Let $\underline{\alpha}_{6}=\max \left\{\underline{\alpha}_{1}, \underline{\alpha}_{2}, \underline{\alpha}_{3}, \underline{\alpha}_{4}, \underline{\alpha}_{5}\right\}$. The above discussion implies that for all $\alpha \in\left[\underline{\alpha}_{6}, 1\right]$, the all$s$ state is uniquely stochastically stable for all models of reference points and all $\lambda \geq 1$.

Case 2: $a_{11} \geq a_{01} \geq a_{00}>a_{10}$. The proof is identical to the previous case. We can find $\underline{\alpha}_{7}$ such that for any $\alpha \in\left[\underline{\alpha}_{7}, 1\right]$, all-s is uniquely stochastically stable for all models of reference points and all $\lambda \geq 1$.

Case 3: $a_{00}>a_{11} \geq a_{01} \geq a_{10}$. In this case, $v\left(a_{00}, r\right)>v\left(a_{11}, r\right)$ and $v\left(a_{01}, r\right) \geq v\left(a_{10}, r\right)$ indicate that $u_{0}\left(\frac{1}{2}, r\right)-u_{1}\left(\frac{1}{2}, r\right)>0$ for any $\lambda \geq 1$ and any $r$.

Proof of Proposition 7.5. Consider a synchronous learning protocol in which every agent receives a revision opportunity with probability $q>0$ in each period. We call this protocol the Independent Learning (IL) protocol. Similarly to the asynchronous protocol, the all-s state is uniquely stochastically stable under the IL protocol if it has a larger basin of attraction than the other strategy (see Kandori and Rob (1998)). Thus, the stochastically stable states under the two protocols coincide. 
Proposition 1 of Alós-Ferrer and Netzer (2015) implies that the all-s state is uniquely stochastically stable under any regular synchronous learning protocol if it is uniquely stochastically stable under the IL protocol and the asynchronous protocol. Hence, the conclusion of Theorem 3.2 must hold for all regular synchronous learning protocols.

\section{Acknowledgments}

The authors sincerely thank an advisory editor and two anonymous referees for the comments and suggestions that greatly improved the paper. We also thank Ned Augenblick, Jonathan Newton, Yasuhiro Shirata, and participants of Central European Program for Economic Theory 2015 Meeting, 2015 East Asian Game Theory Conference, 2015 Economic Science Association North America Meeting, 2016 Japanese Economic Association Fall Meeting, and 2017 Decision Theory Workshop at

Hitotsubashi University for their comments and suggestions. Sawa acknowledges financial support from JSPS Grant-in-Aid 15K17023 and 18K12740.

\section{References}

Alós-Ferrer, C. and Netzer, N. (2015). Robust stochastic stability. Economic Theory, 58:31-57.

Battalio, R., Samuelson, L., and Huyck, J. V. (2001). Optimization incentives and coordination failure in laboratory stag hunt games. Econometrica, 69:749-764.

Battigalli, P. and Dufwenberg, M. (2009). Dynamic psychological games. Journal of Economic Theory, 144:1-35.

Bilancini, E. and Boncinelli, L. (2016). The evolution of conventions under condition-dependent mistakes. Unpublished Manuscript, Università degli Studi di Firenze.

Blume, L. (1993). The statistical mechanics of strategic interaction. Games and Economic Behavior, $5: 387-424$.

Blume, L. E. (2003). How noise matters. Games and Economic Behavior, 44(2):251-271.

Börgers, T. and Sarin, R. (2000). Naive reinforcement learning with endogenous aspirations. International Economic Review, 41:921-950.

Brandts, J. and Cooper, D. J. (2006). A change would do you good .... an experimental study on how to overcome coordination failure in organizations. American Economic Review, 96:669-693. 
Cachon, G. P. and Camerer, C. F. (1996). Loss-avoidance and forward induction in experimental coordination games. Quarterly Journal of Economics, 111:165-194.

Ellison, G. (1993). Learning, local interaction, and coordination. Econometrica, 61:1047-1071.

Ellison, G. (2000). Basins of attraction, long-run stochastic stability, and the speed of step-by-step evolution. Review of Economic Studies, 67:17-45.

Fehr, E., Klein, A., and Schmidt, M. K. (2001). Fairness and contract design. Econometrica, $75: 121-154$.

Fehr, E. and Schmidt, K. M. (1999). A theory of fairness, competition, and cooperation. The Quarterly Journal of Economics, 114:817-868.

Feltovich, N. (2011). The effect of subtracting a constant from all payoffs in a hawk-dove game: Experimental evidence of loss aversion in strategic behavior. Southern Economic Journal, 77:814826.

Feltovich, N., Iwasaki, A., and Oda, S. H. (2012). Payoff levels, loss avoidance, and equilibrium selection in games with multiple equilibria: An experimental study. Economic Inquiry, 50:932952.

Festinger, L. (1954). A theory of social comparison processes. Human Relations, 7:117-140.

Geanakoplos, J., Pearce, D., and Stacchetti, E. (1989). Psychological games and sequential rationality. Games and Economic Behavior, 1:60-79.

Harsanyi, J. C. and Selten, R. (1988). A General Theory of Equilibrium Selection in Games. MIT Press, first edition.

Hopkins, E. and Kornienko, T. (2009). Status, affluence, and inequality: Rank-based comparisons in games of status. Games and Economic Behavior, 67:552-568.

Hwang, S.-H. and Newton, J. (2017). Payoff-dependent dynamics and coordination games. Economic Theory, 64(3):589-604.

Hwang, S.-H. and Rey-Bellet, L. (2017). Positive feedback in coordination games: stochastic evolutionary dynamics and the logit choice rule. arXiv. arXiv:1701.04870v3 [cs.GT]. 
Kahneman, D., Knetsch, J. L., and Thaler, R. H. (1991). Anomalies: The endowment effect, loss aversion, and status quo bias. Journal of Economic Perspectives, 5:193-206.

Kahneman, D. and Tversky, A. (1979). Prospect theory: An analysis of decision under risk. Econometrica, 47:263-292.

Kandori, M., Mailath, G. J., and Rob, R. (1993). Learning, mutation, and long run equilibria in games. Econometrica, 61:1003-1037.

Kandori, M. and Rob, R. (1995). Evolution of equilibria in the long run: A general theory and applications. Journal of Economic Theory, 65:383-414.

Kandori, M. and Rob, R. (1998). Bandwagon effects and long run technology choice. Games and Economic Behavior, 22:30-60.

Knetsch, J. L. and Sinden, J. A. (1984). Willingness to pay and compensation demanded: Experimental evidence of an unexpected disparity in measures of value. Quarterly Journal of Economics, 99:507-521.

Köszegi, B. and Rabin, M. (2006). A model of reference-dependent preferences. Quarterly Journal of Economics, 121:1133-1165.

Kreindler, G. E. and Young, H. P. (2013). Fast convergence in evolutionary equilibrium selection. Games and Economic Behavior, 80:39-67.

Lim, W. and Neary, P. R. (2016). An experimental investigation of stochastic adjustment dynamics. Games and Economic Behavior, 100:208-219.

Maruta, T. (2002). Binary games with state dependent stochastic choice. Journal of Economic Theory, 103:351-376.

Mäs, M. and Nax, H. H. (2016). A behavioral study of noise in coordination games. Journal of Economic Theory, 162:195-208.

Morris, S., Rob, R., and Shin, H. S. (1995). p-dominance and belief potential. Econometrica, $63: 145-157$.

Neary, P. R. (2012). Competing conventions. Games and Economic Behavior, 76(1):301-328. 
Newton, J. (2018a). The deconstruction of conventions. Unpublished Manuscript, Available at SSRN: https://ssrn.com/abstract $=3216269$.

Newton, J. (2018b). Evolutionary game theory: A renaissance. Games, 9(2):31.

Nöldeke, G. and Samuelson, L. (1993). An evolutionary analysis of backward and forward induction. Games and Economic Behavior, 5:425-454.

Norman, T. W. (2009). Rapid evolution under inertia. Games and Economic Behavior, 66:865-879.

Oyama, D., Sandholm, W. H., and Tercieux, O. (2015). Sampling best response dynamics and deterministic equilibrium selection. Theoretical Economics, 10:243-281.

Peski, M. (2010). Generalized risk-dominance and asymmetric dynamics. Journal of Economic Theory, 145(1):216-248.

Rabin, M. (1993). Incorporating fiarness into game theory and economics. American Economic Review, 83:1281-1302.

Rhode, P. and Stegeman, M. (1996). A comment on "learning, mutation, and long-run equilibria in games". Econometrica, 64:443-449.

Rydval, O. and Ortmann, A. (2005). Loss avoidance as selection principle: Evidence from simple stag-hunt games. Economics Letters, 88:101-107.

Samuelson, W. and Zeckhauser, R. (1988). Status quo bias in decision making. Journal of Risk and Uncertainty, 1:7-59.

Sandholm, W. H. (1998). Simple and clever decision rules for a model of evolution. Economics Letters, 61:165-170.

Sandholm, W. H. (2001). Almost global convergence to p-dominant equilibrium. International Journal of Game Theory, 30:107-116.

Sandholm, W. H. (2010). Orders of limits for stationary distributions, stochastic dominance, and stochastic stability. Theoretical Economics, 5:1-26.

Sandholm, W. H. and Staudigl, M. (2016). Large deviations and stochastic stability in the small noise double limit. Theoretical Economics, 11:279-355. 
Sarin, R. and Vahid, F. (1999). Payoff assessments without probabilities: A simple dynamic model of choice. Games and Economic Behavior, 28:249-309.

Sawa, R. (2015). Prospect theory Nash bargaining solution and its stochastic stability. Unpublished manuscript, University of Tsukuba.

Sawa, R. and Wu, J. (2018). Reference-dependent preferences, super-dominance and stochastic stability. Journal of Mathematical Economics (forthcoming).

Schmidt, D., Shupp, R., Walker, J. M., and Ostrom, E. (2003). Playing safe in coordination games: the roles of risk dominance, payoff dominance, and history of play. Games and Economic Behavior, 42:281-299.

Shalev, J. (2000). Loss aversion equilibrium. International Journal of Game Theory, 29:269-287.

Staudigl, M. (2012). Stochastic stability in asymmetric binary choice coordination games. Games and Economic Behavior, 75:372-401.

Staudigl, M. and Weidenholzer, S. (2014). Constrained interactions and social coordination. Journal of Economic Theory, 152:41-63.

Thaler, R. (1980). Toward a positive theory of consumer choice. Journal of Economic Behavior and Organization, 1:39-60.

Tversky, A. and Kahneman, D. (1992). Advances in prospect theory: Cumulative representation of uncertainty. Journal of Risk and Uncertainty, 5:297-323.

Veblen, T. (1899). The Theory of the Leisure Class: An Economic Study in the Evolution of Institutions. Macmillan, first edition.

von Neumann, J. and Morgenstern, O. (1944). Theory of Games and Economic Behavior. Princeton University Press, first edition.

Young, H. P. (1993). The evolution of conventions. Econometrica, 61:57-84. 